\title{
Two meson systems with Ginsparg-Wilson valence quarks
}

\author{
Jiunn-Wei Chen, ${ }^{1, *}$ Donal O'Connell, ${ }^{2, \dagger}$ and André Walker-Loud ${ }^{3,4, *}$ \\ ${ }^{1}$ Department of Physics and Center for Theoretical Sciences, National Taiwan University, Taipei 10617, Taiwan \\ ${ }^{2}$ California Institute of Technology, Pasadena, California 91125, USA \\ ${ }^{3}$ Department of Physics, University of Maryland, College Park, Maryland 20742-4111, USA \\ ${ }^{4}$ Department of Physics, University of Washington, Box 351560, Seattle, Washington 98195-1560, USA
}

(Received 4 December 2006; published 1 March 2007)

\begin{abstract}
Unphysical effects associated with finite lattice spacing and partial quenching may lead to the presence of unphysical terms in chiral extrapolation formulas. These unphysical terms must then be removed during data analysis before physical predictions can be made. In this work, we show that through next-to-leading order, there are no unphysical counterterms in the extrapolation formulas, expressed in lattice-physical parameters, for meson scattering lengths in theories with Ginsparg-Wilson valence quarks. Our work applies to most sea quark discretizations, provided that chiral perturbation theory is a valid approximation. We demonstrate our results with explicit computations and show that, in favorable circumstances, the extrapolation formulas do not depend on the unknown constant $C_{\text {Mix }}$ appearing at lowest order in the mixed action chiral Lagrangian. We show that the $I=1 K K$ scattering length does not depend on $C_{\mathrm{Mix}}$ in contrast to the $I=3 / 2 K \pi$ scattering length. In addition, we show that these observables combined with $f_{K} / f_{\pi}$ and the $I=2 \pi \pi$ scattering length share only two linearly independent sets of counterterms, providing a means to test the mixed action theory at one lattice spacing. We therefore make a prediction for the $I=1 K K$ scattering length.
\end{abstract}

DOI: 10.1103/PhysRevD.75.054501

PACS numbers: $12.38 . \mathrm{Gc}$

\section{INTRODUCTION}

There is currently a tension in lattice simulations of QCD phenomena between the need for quarks obeying chiral symmetry on the lattice and the need for quark masses light enough that one is in the chiral regime. This tension occurs because quark discretization schemes which obey chiral symmetry on the lattice, such as domain wall fermions [1-3] or overlap fermions [4-6], both of which satisfy the Ginsparg-Wilson relation [7,8], are numerically expensive to simulate. On the other hand, Wilson fermions [9] or staggered fermions $[10,11]$ are faster but violate chiral symmetry at nonzero lattice spacing.

One way of resolving this tension is to recognize that the most computationally intensive stage of a fully dynamical simulation is the evaluation of the quark determinant. This determinant is associated with the sea quarks and is a component of the probability measure on the space of gauge field configurations. This observation has long been the motivation for partial quenching (PQ) $[12,13]-$ sea quark masses are taken to be larger than valence quark masses so that the sea quarks are more localized and the determinant is easier to compute. The notion of a "mixed action" (MA) simulation takes this line of reasoning one step farther [14,15]. A mixed action simulation uses different quark discretizations in the sea and valence sectors. In this case, the valence quarks can be chosen to obey the Ginsparg-Wilson relation so that they enjoy chiral symmetry at finite lattice spacing. The numerically

\footnotetext{
*Electronic address: jwc@ @ phys.ntu.edu.tw

${ }^{\dagger}$ Electronic address: donal@theory.caltech.edu

‡Electronic address: walkloud@umd.edu
}

expensive sea quarks, on the other hand, can be chosen to be inexpensive Wilson or (rooted) staggered sea quarks, for example.

There recently have been a significant number of mixed action lattice QCD simulations, [16-26], the majority of which have employed domain wall valence fermions on the publicly available MILC lattices [27]. ${ }^{1}$ The effective theories appropriate for mixed action simulations were originally developed in Refs. [14,15] for Ginsparg-Wilson (GW) valence fermions on Wilson sea fermions and later for $\mathrm{GW}$ valence fermions on staggered sea fermions [30]; these theories have received considerable theoretical attention recently [31-36] in response to the numerical interest.

In this article, we study aspects of the chiral perturbation theories appropriate for mesonic processes in mixed action simulations with Ginsparg-Wilson valence quarks. The chiral properties of the Ginsparg-Wilson valence quarks are central to our work, so we adopt the convention that when we refer to a mixed action simulation, we imply that the valence quarks are Ginsparg-Wilson. We work consistently at next-to-leading order (NLO) in the effective field theory expansion, which is a dual expansion in powers of the quark mass $m_{q}$ and the lattice spacing $a$. At this order, one can view current lattice simulations as being methods of computing the values of certain coefficients in the NLO chiral Lagrangian, which for mesonic quantities is known as the Gasser-Leutwyler Lagrangian [37,38]. This is because lattice simulations are performed at quark masses larger than the physical quark mass, so that lattice data

\footnotetext{
${ }^{1}$ The MILC lattices themselves utilize asqtad-improved $[28,29]$, staggered sea fermions.
} 
must be fit to formulas computed in chiral perturbation theory. These fits determine the unknown coefficients occurring in the chiral formulas, known as low energy constants, so that the chiral expression can then be used at the physical values of the meson masses and decay constants to predict the results of physical experiments. Frequently there are nonphysical operators in the NLO chiral Lagrangians describing discretized fermions. For example, there are of order 100 operators in the NLO staggered chiral Lagrangian [39] compared to order 10 in the Gasser-Leutwyler Lagrangian. These unphysical operators lead to unphysical terms in chiral extrapolation formulas which must somehow be removed to make physical predictions. One might think that this will also be an issue in mixed action chiral perturbation theory (MA $\chi \mathrm{PT})$, since there are certainly many additional operators at NLO. However, we show that mixed action lattice simulations of mesonic scattering lengths do not depend on any unphysical operators at NLO, if these scattering lengths are expressed in terms of the pion mass measured on the lattice and the decay constant measured on the lattice [40]. For linguistic brevity, we will refer to the pion mass measured on the lattice as the lattice-physical pion mass and similarly for the decay constant.

Each choice of sea quark discretization leads to a different MA $\chi$ PT. For example, tree level shifts of the masses of mesons composed of two sea quarks, or of one valence and one sea quark, are different for staggered sea quarks and for Wilson sea quarks. Therefore, one may think that the chiral extrapolation formulas depend on the nature of the sea quark discretization. We show that, at NLO, the only difference between the extrapolation formulas is in the leading order mass shifts of the mesons composed of two sea quarks. Thus, once these mass shifts are known, one can use the same extrapolation formulas for different sea quark discretizations. ${ }^{2}$ In fact, any sea quark discretization will do provided, ${ }^{3}$ first, that QCD is recovered in the continuum limit, and second, that the sea-sea mesons may be described at leading order by chiral perturbation theory with the usual kinetic and mass terms, or that the nonlocality of the appropriate chiral perturbation theory is correctly captured by the replica method [42]. ${ }^{4}$ In addition, we have assumed that the quarks of the sea sector are only distinguished by their masses, so that, for example, the same discretization scheme has been used for all sea

\footnotetext{
${ }^{2}$ For staggered fermions, these mass corrections to the mesons are well know [41]. However, these effects are less well determined for Wilson fermions.

${ }^{3}$ In this paper, we restrict ourselves to isospin symmetric masses in the valence and sea sectors.

${ }^{4}$ We have in mind the current discussion regarding whether rooted staggered fermions become QCD in the continuum limit. There is growing numerical and formal evidence lending support to the hope that rooted staggered fermions are in the same universality class as QCD. We refer the reader to Ref. [43] for current summary of the issues.
}

quarks, and we assume that chiral perturbation theory itself is a valid approximation.

There are still various challenges facing mixed action simulations. Mixed action simulations always violate unitarity at finite lattice spacing. ${ }^{5}$ In $\mathrm{MA} \chi \mathrm{PT}$ and $\mathrm{PQ} \chi \mathrm{PT}$, the most severe unitarity violations are encoded in hairpin propagators of flavor-neutral mesons. We point out a simple parametrization which allows a convenient bookkeeping of these unitarity violating effects. Additionally, the value of the new constant $C_{\mathrm{Mix}}$, that appears in the $\mathrm{LO}$ mixed action Lagrangian, is currently unknown. This term leads to an additive lattice spacing dependent mass shift of "mixed" mesons consisting of one valence and one sea quark. This causes a mismatch of the meson masses composed of different quarks but does not play a role in the well-known enhanced chiral logarithms [13,44,45] or the enhanced power-law volume dependence of two-hadron states [46]. ${ }^{6}$ In addition, the value of this constant is presumably different for each sea quark discretization. However, we show that under favorable circumstances physical quantities such as scattering lengths do not depend on this constant, as was first noticed in Ref. [34].

Finally, to demonstrate our arguments, we determine various NLO formulas for use in chiral extrapolation of certain mesonic quantities. We have computed the $K K$ and $K \pi$ scattering lengths in $S U(6 \mid 3) \mathrm{MA} \chi \mathrm{PT}$. In addition, for completeness, we present the $\pi \pi$ scattering length in $S U(4 \mid 2)$ and $S U(6 \mid 3) \mathrm{MA} \chi \mathrm{PT}$, which were computed in Ref. [34] as well as the $\pi$ and $K$ meson masses and decay constants, which were first computed in Refs. [14,15,30], but we express these quantities in terms of the PQ parameters we introduce in Sec. II A. We conclude with a discussion of our results, and, in particular, show that among the three meson scattering lengths mentioned above and the quantity $f_{K} / f_{\pi}$ there are only two linearly independent counterterms at NLO, which are the corresponding physical counterterms of $\chi \mathrm{PT}$. Therefore, these four processes provide a means to test the MA formalism with only one lattice spacing. In the appendices we collect the various formulas which are necessary for the chiral extrapolations of the quantities we discuss in this paper.

\footnotetext{
${ }^{5}$ For arbitrarily small lattice spacings, the differences arising from the different lattice actions will become negligible, which practically means smaller than the statistical and systematic uncertainties for a given observable, and these unitarity violating terms will no longer be important (assuming the quark masses are tuned equal). This of course also implies that a MA effective field theory description will no longer be necessary; however, for MA lattice simulations today and the foreseeable future, $\mathrm{MA} \chi \mathrm{PT}$ is the necessary tool for controlling extrapolations to the physical point.

${ }^{6}$ In this article we do not discuss the observed negative norm issues involving scalar meson correlators $[41,47]$, but these can at least be qualitatively understood with the appropriate effective field theory methods $[48,49]$.
} 


\section{MIXED ACTION EFFECTIVE FIELD THEORY}

We will not give a thorough introduction to mixed action or partially quenched theories here. We will simply give a brief review to introduce our notation and power counting. For a good introduction to MA theories we refer the reader to Refs. $[14,15,30]$, and for PQ theories to Refs. [50,51].

\section{A. Mixed actions at lowest order}

To construct the appropriate Lagrangian to a given order, one must specify a power counting. As mentioned above, $\chi \mathrm{PT}$ is a systematic expansion about the zero momentum, zero quark mass limit, for which the small expansion parameter is

$$
\varepsilon_{m}^{2} \sim \frac{p^{2}}{\Lambda_{\chi}^{2}} \sim \frac{m_{\pi}^{2}}{\Lambda_{\chi}^{2}}
$$

where $m_{\pi}^{2} \propto m_{q}$. For effective theories extended to include lattice spacing artifacts, one must include an additional small parameter. ${ }^{7}$ We will be interested in theories for which the leading sea quark lattice spacing dependence is $\mathcal{O}\left(a^{2}\right)$, such as staggered, $\mathcal{O}(a)$-improved Wilson [55], twisted mass at maximal twist [56], or chiral fermions. Therefore, we shall denote the small parameter counting lattice spacing artifacts to be

$$
\varepsilon_{a}^{2} \sim a^{2} \Lambda_{\mathrm{QCD}}^{2}
$$

and we shall work consistently in the dual expansion to

$$
\mathcal{O}\left(\varepsilon_{m}^{4}\right), \quad \mathcal{O}\left(\varepsilon_{m}^{2} \varepsilon_{a}^{2}\right), \quad \mathcal{O}\left(\varepsilon_{a}^{4}\right) .
$$

At leading order (LO) in the quark mass expansion, the mixed action Lagrangian is simply given by the partially quenched Lagrangian [14],

$$
\mathcal{L}=\frac{f^{2}}{8} \operatorname{str}\left(\partial_{\mu} \Sigma \partial^{\mu} \Sigma^{\dagger}\right)+\frac{f^{2} B_{0}}{4} \operatorname{str}\left(m_{q} \Sigma^{\dagger}+\Sigma m_{q}^{\dagger}\right),
$$

where we use the normalization $f \simeq 132 \mathrm{MeV}$, and

$$
\Sigma=\exp \left(\frac{2 i \Phi}{f}\right), \quad \Phi=\left(\begin{array}{cc}
M & \chi^{\dagger} \\
\chi & \tilde{M}
\end{array}\right)
$$

The matrices $M$ and $\tilde{M}$ contain bosonic mesons while $\chi$ and $\chi^{\dagger}$ contain fermionic mesons with one ghost quark or antiquark. To be specific, we will discuss the theory with three valence (and ghost) quarks and three sea quarks, for which $^{8}$

\footnotetext{
${ }^{7}$ The general procedure [52] is to construct the continuum Symanzik quark level effective theory for a given lattice action $[53,54]$ and then build the low-energy effective theory with spurion analysis on this continuum lattice action.

${ }^{8}$ For staggered sea quarks, each sea quark label implicitly includes a taste label as well. For example, $\phi_{u j}$ is a $1 \times 4$ vector in taste-space.
}

$$
\begin{aligned}
M & =\left(\begin{array}{llllll}
\eta_{u} & \pi^{+} & K^{+} & \phi_{u j} & \phi_{u l} & \phi_{u r} \\
\pi^{-} & \eta_{d} & K^{0} & \phi_{d j} & \phi_{d l} & \phi_{d r} \\
K^{-} & \bar{K}^{0} & \eta_{s} & \phi_{s j} & \phi_{s l} & \phi_{s r} \\
\phi_{j u} & \phi_{j d} & \phi_{j s} & \eta_{j} & \phi_{j l} & \phi_{j r} \\
\phi_{l u} & \phi_{l d} & \phi_{l s} & \phi_{l j} & \eta_{l} & \phi_{l r} \\
\phi_{r u} & \phi_{r d} & \phi_{r s} & \phi_{r j} & \phi_{r l} & \eta_{r}
\end{array}\right), \\
\tilde{M} & =\left(\begin{array}{lll}
\tilde{\eta}_{u} & \tilde{\pi}^{+} & \tilde{K}^{+} \\
\tilde{\pi}^{-} & \tilde{\eta}_{d} & \tilde{K}^{0} \\
\tilde{K}^{-} & \tilde{\bar{K}}^{0} & \tilde{\eta}_{s}
\end{array}\right), \\
\chi^{\dagger} & =\left(\begin{array}{llll}
\phi_{u \tilde{u}} & \phi_{u \tilde{d}} & \phi_{u \tilde{s}} \\
\phi_{d \tilde{u}} & \phi_{d \tilde{d}} & \phi_{d \tilde{s}} \\
\phi_{s \tilde{u}} & \phi_{s \tilde{d}} & \phi_{s \tilde{s}} \\
\phi_{j \tilde{u}} & \phi_{j \tilde{d}} & \phi_{j \tilde{s}} \\
\phi_{l \tilde{u}} & \phi_{l \tilde{d}} & \phi_{l \tilde{s}} \\
\phi_{r \tilde{u}} & \phi_{r \tilde{d}} & \phi_{r \tilde{s}}
\end{array}\right) .
\end{aligned}
$$

The upper $N_{v} \times N_{v}$ block of $M$ contains the usual mesons composed of a valence quark and antiquark. The lower $N_{s} \times N_{s}$ block of $M$ contains the sea quark-antiquark mesons and the off-diagonal block elements of $M$ contain bosonic mesons of mixed valence-sea type.

For MA theories there are two types of operators we need to consider at LO in $\varepsilon_{a}^{2}$. There are those which modify the sea-sea sector meson potential, which we shall denote as $U_{\text {sea }}$, and those which modify the mixed meson potential, which we shall denote as $\mathcal{U}_{V S}$, such that the Lagrangian, Eq. (4), is modified by the additional terms (following the sign conventions of Ref. [30]),

$$
\mathcal{L}_{\mathrm{MA}}=-a^{2}\left(\mathcal{U}_{\mathrm{sea}}-\mathcal{U}_{V S}\right) .
$$

We shall not specify the form of the sea-sea meson potential $\mathcal{U}_{\text {sea }}$, but only note again that at the order we are concerned with, we only need to know how the masses of the sea-sea mesons are modified at LO in $\varepsilon_{a}^{2}$ which have been discussed, for example, in Refs. [14,30]. The other important thing to know is that the structure of $\mathcal{U}_{V S}$ is independent of the type of sea quark and is given by $[14,30]$

$$
\mathcal{U}_{V S}=C_{\text {Mix }} \operatorname{str}\left(T_{3} \Sigma T_{3} \Sigma^{\dagger}\right)
$$

where the flavor matrix $T_{3}$ is a difference in projectors onto the valence and sea sectors of the theory,

$$
T_{3}=\mathcal{P}_{S}-\mathcal{P}_{V}=\operatorname{diag}\left(-I_{V}, I_{S},-I_{V}\right) .
$$

This operator leads to an additive shift of the valence-sea meson masses, such that all the pseudo-Goldstone mesons composed of either valence quarks $v$, sea quarks $s$, or both have LO masses given by ${ }^{9}$

\footnotetext{
${ }^{9}$ Here and throughout this article, we use tildes over the masses to indicate additive lattice spacing corrections to the meson masses.
} 


$$
\begin{aligned}
m_{v_{1} v_{2}}^{2} & =B_{0}\left(m_{v_{1}}+m_{v_{2}}\right), \\
\tilde{m}_{v s}^{2} & =B_{0}\left(m_{v}+m_{s}\right)+a^{2} \Delta_{\text {Mix }}, \\
\tilde{m}_{s_{1} s_{2}}^{2} & =B_{0}\left(m_{s_{1}}+m_{s_{2}}\right)+a^{2} \Delta_{\text {sea }},
\end{aligned}
$$

with $\Delta_{\text {sea }}$ determined by $\mathcal{U}_{\text {sea }}$ and ${ }^{10}$

$$
\Delta_{\mathrm{Mix}}=\frac{16 C_{\mathrm{Mix}}}{f^{2}} .
$$

The most severe and well-known unitarity violating feature of PQ and MA theories is the presence of double pole propagators in the flavor-neutral mesons [12]. In particular the momentum space propagators between two mesons composed of valence quarks of flavors $a$ and $b$ respectively are given by ${ }^{11}$

$$
\begin{aligned}
\mathcal{G}_{a b}\left(p^{2}\right)= & \frac{i \delta_{a b}}{p^{2}-m_{a a}^{2}} \\
& -\frac{i}{3} \frac{\left(p^{2}-\tilde{m}_{j j}^{2}\right)\left(p^{2}-\tilde{m}_{r r}^{2}\right)}{\left(p^{2}-m_{a a}^{2}\right)\left(p^{2}-m_{b b}^{2}\right)\left(p^{2}-\tilde{m}_{X}^{2}\right)}
\end{aligned}
$$

where $\tilde{m}_{X}$ is the mass of the $\eta_{\text {sea }}$-field,

$$
\tilde{m}_{X}^{2}=\frac{1}{3} \tilde{m}_{j j}^{2}+\frac{2}{3} \tilde{m}_{r r}^{2}
$$

When the valence quark masses are equal, either in the isospin limit of light quarks or for the same flavor, $a=b$, the above propagator acquires a double pole. It is these double poles which lead to the well-known sicknesses of PQ $\chi$ PT, such as the enhanced chiral logs $[13,44,45]$ and enhanced power-law volume dependence of two-particle states [46]. Here, we introduce what we call "partial quenching parameters," which are a difference in the quark masses for PQ theories and, more generally for MA theories, a difference in the masses of mesons composed of two sea quarks and two valence quarks. For PQ theories, when these quantities are zero, the theory reduces to an unquenched theory. For MA theories, when one tunes these parameters to zero, one tunes the double pole structure of the flavor-neutral meson propagators to zero up to higher order corrections, and thus has the most QCD-like scenario for a MA theory [we note that there is still a mismatch in

\footnotetext{
${ }^{10}$ For a twisted mass sea [56], one must keep separate track of the neutral and charged mesons as they receive a relative $\mathcal{O}\left(a^{2}\right)$ splitting [57], similar to the various taste mesons for staggered fermions.

${ }^{11}$ In mixed action theories, there are additional hairpin interactions proportional to the lattice spacing which arise from unphysical operators in the theory, similar to the lattice spacing dependent hairpin interactions in staggered $\chi \mathrm{PT}[58]$. For $\mathcal{O}(a)$ improved Wilson fermions and staggered fermions, these effects are higher order than we are concerned with in this paper [32], which is also true for twisted mass fermions at maximal twist. For Wilson and twisted mass fermions (away from maximal twist), these effects appear at the order we are working and must be included. We assume for the rest of the paper that the sea quark scaling violations are $\mathcal{O}\left(a^{2}\right)$ or higher.
}

the mass of the mixed meson, composed of one valence and one sea quark, Eq. (10), from the others]. We therefore introduce the partial quenching parameters, ${ }^{12}$

$$
\begin{gathered}
\tilde{\Delta}_{j u}^{2} \equiv \tilde{m}_{j j}^{2}-m_{u u}^{2}=2 B_{0}\left(m_{j}-m_{u}\right)+a^{2} \Delta_{\text {sea }}+\ldots, \\
\tilde{\Delta}_{r s}^{2} \equiv \tilde{m}_{r r}^{2}-m_{s s}^{2}=2 B_{0}\left(m_{r}-m_{s}\right)+a^{2} \Delta_{\text {sea }}+\ldots,
\end{gathered}
$$

where the dots denote higher order corrections to the meson masses. We will now move on to discuss the general structure of mixed action theories for arbitrary sea quarks at the next order.

\section{B. Mixed action $\chi \mathrm{PT}$ at NLO}

It was shown in Ref. [34] that the $I=2 \pi \pi$ scattering length at NLO, expressed in terms of the bare parameters of the chiral Lagrangian, is ${ }^{13}$

$$
\begin{aligned}
m_{\pi} a_{\pi \pi}^{I=2}= & -\frac{m_{u u}^{2}}{8 \pi f^{2}}\left\{1+\frac{m_{u u}^{2}}{(4 \pi f)^{2}}\left[4 \ln \left(\frac{m_{u u}^{2}}{\mu^{2}}\right)\right.\right. \\
& \left.+4 \frac{\tilde{m}_{j u}^{2}}{m_{u u}^{2}} \ln \left(\frac{\tilde{m}_{j u}^{2}}{\mu^{2}}\right)-1+\ell_{\pi \pi}^{\prime}(\mu)\right] \\
& -\frac{m_{u u}^{2}}{(4 \pi f)^{2}}\left[\frac{\tilde{\Delta}_{j u}^{4}}{6 m_{u u}^{4}}+\frac{\tilde{\Delta}_{j u}^{2}}{m_{u u}^{2}}\left[\ln \left(\frac{m_{u u}^{2}}{\mu^{2}}\right)+1\right]\right] \\
& \left.+\frac{\tilde{\Delta}_{j u}^{2}}{(4 \pi f)^{2}} \ell_{\mathrm{PQ}}^{\prime}(\mu)+\frac{a^{2}}{(4 \pi f)^{2}} \ell_{a^{2}}^{\prime}(\mu)\right\} .
\end{aligned}
$$

Let us point out some features of this expression which are relevant from the point of view of chiral extrapolations. Equation (15) depends on the mass $\tilde{m}_{j u}$ of a mixed valencesea meson and consequently the expression depends on the value of the parameter $C_{\mathrm{Mix}}$. In addition, there is a dependence on the unphysical unknowns $\ell_{\mathrm{PQ}}^{\prime}(\mu)$ and $\ell_{a^{2}}^{\prime}(\mu)$, as well as the decay constant and chiral condensate in the chiral limit, $f$ and $B_{0}$. Thus, Eq. (15) depends on three unphysical unknown parameters and three physical unknown parameters, $\ell_{\pi \pi}^{\prime}(\mu), f$, and $B_{0}$. One must fit all unknown parameters to extrapolate lattice data but only three are of intrinsic interest.

In terms of lattice-physical parameters, the same scattering length becomes

$$
\begin{aligned}
m_{\pi} a_{\pi \pi}^{I=2}= & -\frac{m_{\pi}^{2}}{8 \pi f_{\pi}^{2}}\left\{1+\frac{m_{\pi}^{2}}{\left(4 \pi f_{\pi}\right)^{2}}\left[3 \ln \left(\frac{m_{\pi}^{2}}{\mu^{2}}\right)-1\right.\right. \\
& \left.\left.-l_{\pi \pi}^{I=2}(\mu)-\frac{\tilde{\Delta}_{j u}^{4}}{6 m_{\pi}^{4}}\right]\right\} .
\end{aligned}
$$

\footnotetext{
${ }^{12}$ For a staggered sea it is the taste-identity meson masses which enter these PQ parameters [30] (which have been measured [41]) while for a twisted mass sea, it is the neutral pion mass.

${ }^{13}$ Here, we show the scattering length for a two-sea flavor theory. In the appendix, we also list the result for the three-sea flavor theory. However, the following discussion of the counterterm structure of the NLO Lagrangian is independent of the number of sea flavors.
} 
Notice that this expression does not depend on the mixed valence-sea mesons, and, in fact, the only unknown terms in the expression is the physical parameter $\ell_{\pi \pi}(\mu)$ and the sea-sea meson mass shift in $\tilde{\Delta}_{j u}^{2}$, Eq. (10), which is already determined for staggered sea quarks. Thus, chiral extrapolations using the formula Eq. (16) requires fitting only one parameter (two for nonstaggered sea quarks), in contrast to chiral extrapolations using the scattering length expressed in terms of the bare parameters, Eq. (15). Our goal in this section is to understand the origin of this simplification and under what circumstances we may expect similar simplifications to occur in other processes. To do so, we must discuss the structure of the NLO terms of the MA $\chi \mathrm{PT}$ Lagrangian.

The symmetry structure of the underlying mixed action form of QCD determines the NLO operators in the mixed action chiral Lagrangian through a spurion analysis. However, the symmetries enjoyed by the valence quarks are different to the symmetries of the sea quarks in a mixed action theory. In particular, we only consider GW valence quarks which have a chiral symmetry; the numerically cheaper sea quarks typically violate chiral symmetry. Thus, it is helpful to consider spurions arising from the valence sector separately to the spurions of the sea sector.

The valence sector only violates chiral symmetry explicitly through the quark mass. Therefore, at $\mathrm{NLO},{ }^{14}$ the purely valence spurions are identical to the spurions in continuum, unquenched chiral perturbation theory, and so the valence-valence sector of the NLO mixed action chiral Lagrangian is the Gasser-Leutwyler Lagrangian. The sea sector is different. At finite lattice spacing, the sea sector has enhanced sources of chiral symmetry violation - for example, there are additional spurions associated with taste violation if the sea quarks are staggered, or in the case of a Wilson sea, the Wilson term violates chiral symmetry. Consequently, there are additional spurions in the sea sector. Of course, these spurions must involve the sea quarks and must vanish when the sea quark fields vanish. ${ }^{15}$ Nevertheless, scattering amplitudes expressed in terms of

\footnotetext{
${ }^{14}$ Lattice artifacts such as Lorentz symmetry violation lead to the presence of unphysical operators in the chiral Lagrangian. These operators will not be important in the following, as they are higher order in the chiral expansion for mesons [15] (however they are relevant at $\mathcal{O}\left(\varepsilon_{a}^{2}\right)$ for baryons [31]).

${ }^{15}$ Not all the lattice spacing dependence may be captured with spurion analysis. There are $\mathcal{O}\left(a^{2}\right)$ operators at the quark level which do not break chiral symmetry, for example, $\mathcal{O}^{(6)}=$ $a^{2} \bar{Q} \not D^{3} Q$. This operator leads to an $a^{2}$ renormalization of all the low energy constants of the low-energy theory. Because this operator does not break any of the continuum QCD symmetries, it cannot be distinguished through spurion analysis [15]. Of course, an operator of this form is present when the QCD Lagrangian is run from a high scale (say, the weak scale) down to the scale of the lattice, so its effects could in principle by accounted for by performing a perturbative matching computation between the QCD effective Lagrangian at the scale $\mu=$ $a^{-1}$ and the lattice action.
}

lattice-physical parameters do not explicitly depend on the lattice spacing $a$, as we will now discuss.

In this paper, we work consistently to NLO in the $\mathrm{MA} \chi \mathrm{PT}$ power counting which we have defined in Eq. (3). At this order, the NLO operators in the Lagrangian are only used as counterterms; that is, at NLO one only computes at tree level with the NLO operators. Since the in/out states used in lattice simulations involve purely valence quarks, we must project the NLO operators onto the purely valence quark sector of the theory. Consequently, all of the spurions which involve the sea quark fields vanish. Since the remaining spurions involve the valence quarks alone, we only encounter the symmetry structure of the valence quarks as far as the NLO operators are concerned. These spurions only depend on quark masses and the quark condensate itself, and so there can be no dependence on lattice discretization effects arising in this way. The exception to this argument arises in the case of double trace operators in the NLO chiral Lagrangian; in these cases the valence and sea sectors interact in a flavor-disconnected manner, unlike the operator in Eq. (8). If one trace involves a valence-valence spurion while the other involves a sea-sea spurion, then the trace over the sea may still contribute to a physical quantity, for example, the meson masses and decay constants, see Appendices A, B, and C for explicit examples. Note that the valence-valence operators which occur in these double trace operators must be proportional to one of the two operators present in the LO chiral Lagrangian, Eq. (4). Thus for meson scattering processes, the dependence upon the sea quarks from these double trace operators can only involve a renormalization of the leading order quantities $f$ and $B_{0}$. Both the explicit sea quark mass dependence and the explicit lattice spacing dependence are removed from the scattering processes expressed in terms of the lattice-physical parameters since they are eliminated in favor of the decay constants and meson masses which can simply be measured on the lattice. We therefore conclude that when expressed in lattice-physical parameters, there can be no dependence upon the sea quark masses leading to unphysical PQ counterterms and similarly there can be no dependence upon an unphysical lattice spacing counterterm.

Let us present another more physically intuitive argument concerning the absence of sea quark mass dependence in meson scattering processes. To do so, we must digress briefly on $\pi \pi$ scattering in $S U(3)$ chiral perturbation theory. The strange quark mass $m_{s}$ is a parameter of $S U(3) \chi \mathrm{PT}$, and so one would expect that the $\pi \pi$ scattering length includes analytic terms involving $m_{s}$. However, we can consider a theory in which the strange quark is heavy, so that we may integrate it out; we must then recover $S U(2) \chi \mathrm{PT}$. Chiral symmetry forces any $m_{s}$ dependence in the analytic terms of the $\pi \pi$ amplitude to occur in the form $m_{\pi}^{2} m_{K}^{2}$. But the only counterterm in the 
on-shell $S U(2)$ scattering amplitude [Eq. (16) with $\tilde{\Delta}_{j u}=$ 0 ] is proportional to $m_{\pi}^{4}$. It is not possible to absorb $m_{\pi}^{2} m_{K}^{2}$ into $m_{\pi}^{4}$, so there can be no $m_{s}$ dependence in the $S U(3)$ $\pi \pi$ scattering amplitude. This is indeed the case, as was observed in Ref. [59].

Now, let us return to the PQ and MA theories. For the purposes of this discussion, we can ignore the flavorneutral and ghost sectors, reducing our theory from a $S U(6 \mid 3)$ theory to a $S U(6)$ theory. The sea quark dependence of this $S U(6)$ chiral perturbation theory is analogous to the $m_{s}$ dependence of $S U(3) \chi \mathrm{PT}$ in our $\pi \pi$ scattering example (as in this process the strange quark of $S U(3)$ only participates as a sea quark). A similar decoupling argument tells us that the sea quark masses cannot affect processes involving the valence sector provided one uses the analogues of on-shell parameters which are the latticephysical parameters. We conclude that there can be no analytic dependence on the sea quark masses in a mesonic scattering amplitude. Further, these arguments only depend upon the chiral symmetry of the valence quarks and thus also apply to the lattice spacing dependence.

Now, we shall make these arguments concrete by explicit computations. The NLO Lagrangian describing the valence and sea quark mass dependence is the GasserLeutwyler Lagrangian with traces replaced by supertraces:

$$
\begin{aligned}
\mathcal{L}_{\mathrm{GL}}= & L_{1}\left[\operatorname{str}\left(\partial_{\mu} \Sigma \partial^{\mu} \Sigma^{\dagger}\right)\right]^{2} \\
& +L_{2} \operatorname{str}\left(\partial_{\mu} \Sigma \partial_{\nu} \Sigma^{\dagger}\right) \operatorname{str}\left(\partial^{\mu} \Sigma \partial^{\nu} \Sigma^{\dagger}\right) \\
& +L_{3} \operatorname{str}\left(\partial_{\mu} \Sigma \partial^{\mu} \Sigma^{\dagger} \partial_{\nu} \Sigma \partial^{\nu} \Sigma^{\dagger}\right) \\
& +2 B_{0} L_{4} \operatorname{str}\left(\partial_{\mu} \Sigma \partial^{\mu} \Sigma^{\dagger}\right) \operatorname{str}\left(m_{q} \Sigma^{\dagger}+\Sigma m_{q}^{\dagger}\right) \\
& +2 B_{0} L_{5} \operatorname{str}\left[\partial_{\mu} \Sigma \partial^{\mu} \Sigma^{\dagger}\left(m_{q} \Sigma^{\dagger}+\Sigma m_{q}^{\dagger}\right)\right] \\
& +4 B_{0}^{2} L_{6}\left[\operatorname{str}\left(m_{q} \Sigma^{\dagger}+\Sigma m_{q}^{\dagger}\right)\right]^{2} \\
& +4 B_{0}^{2} L_{7}\left[\operatorname{str}\left(m_{q}^{\dagger} \Sigma-\Sigma^{\dagger} m_{q}\right)\right]^{2} \\
& +4 B_{0}^{2} L_{8} \operatorname{str}\left(m_{q} \Sigma^{\dagger} m_{q} \Sigma^{\dagger}+\Sigma m_{q}^{\dagger} \Sigma m_{q}^{\dagger}\right) .
\end{aligned}
$$

Having a concrete expression for the Lagrangian, ${ }^{16}$ we can easily show explicitly how the sea quark mass dependence disappears. The key is that when constructing NLO correlation functions of purely valence quarks, we can replace the mesonic matrix $\Phi$ in the NLO Lagrangian by a projected matrix

$$
\Phi \rightarrow P_{V} \Phi P_{V}
$$

\footnotetext{
${ }^{16}$ The generators of the PQ and MA theories form graded groups and therefore lack the Cayley-Hamilton identities of $S U(N)$ theories. Therefore, PQ and MA theories have additional operators compared to their $\chi \mathrm{PT}$ counterparts. For example, the $\mathcal{O}\left(p^{4}\right)$ Lagrangian has one additional operator as compared to the Gasser-Leutwyler Lagrangian. However, we do not need to consider the effects of this operator in our analysis as it has been shown that it can be constructed such that it does not contribute to valence quantities until $\mathcal{O}\left(p^{6}\right)$ [60]. This is not generally the case, as is demonstrated by various examples in the baryon sector $[61-70]$.
}

where $P_{V}$ is the projector onto the valence subspace. Therefore, the matrix $\Sigma$ has an expansion of the form

$$
\Sigma=1+P_{V} \Phi P_{V}+\cdots \text {. }
$$

Now, insert this expression into Eq. (17), and consider only the terms involving nonzero powers of $\Phi$. In the single trace operators, the projectors remove any dependence on the sea quark masses. There is still sea quark mass dependence remaining in the double trace operators proportional to $L_{4}$ and $L_{6}$ given by

$$
\begin{aligned}
\delta \mathcal{L}_{\mathrm{GL}}= & 4 B_{0} L_{4} \operatorname{str}\left(\partial_{\mu} \Sigma P_{V} \partial^{\mu} \Sigma^{\dagger} P_{V}\right) \operatorname{str}\left(m_{q}\right) \\
& +16 B_{0}^{2} L_{6} \operatorname{str}\left(m_{q} \Sigma^{\dagger} P_{V}+P_{V} \Sigma m_{q}^{\dagger}\right) \operatorname{str}\left(m_{q}\right) .
\end{aligned}
$$

However, these operators simply shift $f$ and $B_{0}$

$$
\begin{gathered}
f^{2} \rightarrow f^{2}+32 L_{4} B_{0} \operatorname{str}\left(m_{q}\right), \\
f^{2} B_{0} \rightarrow f^{2} B_{0}+64 L_{6} B_{0}^{2} \operatorname{str}\left(m_{q}\right) .
\end{gathered}
$$

Since the parameters $f$ and $B_{0}$ are eliminated in latticephysical parameters in favor of the measured decay constants and meson masses, we can remove the dependence of scattering lengths on the sea quark masses by working in lattice-physical parameters. In an analogous way, we can remove all the explicit lattice spacing dependence. The general MA Lagrangian involving valence-valence external states at $\mathcal{O}\left(\varepsilon_{m}^{2} \varepsilon_{a}^{2}\right)$ can be reduced to the following form:

$$
\begin{aligned}
\delta \mathcal{L}_{\mathrm{MA}}= & a^{2} L_{a^{2}}^{\partial} \operatorname{str}\left(\partial_{\mu} \Sigma P_{V} \partial^{\mu} \Sigma^{\dagger} P_{V}\right) \\
& \times \operatorname{str}\left[f\left(P_{S} \Sigma P_{S}\right) f^{\prime}\left(P_{S} \Sigma^{\dagger} P_{S}\right)\right] \\
& +a^{2} L_{a^{2}}^{m_{q}} \operatorname{str}\left(m_{q} P_{V} \Sigma^{\dagger} P_{V}+P_{V} \Sigma P_{V} m_{q}^{\dagger}\right) \\
& \times \operatorname{str}\left[g\left(P_{S} \Sigma P_{S}\right) g^{\prime}\left(P_{S} \Sigma^{\dagger} P_{S}\right)\right]+\text { H.c. },
\end{aligned}
$$

where the $f$ 's and $g$ 's are functions dependent upon the sea quark lattice action. These then lead to renormalizations of the LO constants,

$$
\begin{aligned}
f^{2} & \rightarrow f^{2}+8 a^{2} L_{a^{2}}^{\partial} \operatorname{str}\left[f\left(P_{S} \Sigma P_{S}\right) f^{\prime}\left(P_{S} \Sigma^{\dagger} P_{S}\right)\right], \\
f^{2} B_{0} & \rightarrow f^{2} B_{0}+4 a^{2} L_{a^{2}}^{m_{q}} \operatorname{str}\left[g\left(P_{S} \Sigma P_{S}\right) g^{\prime}\left(P_{S} \Sigma^{\dagger} P_{S}\right)\right],
\end{aligned}
$$

and just as with the sea quark mass dependence, expressing physical quantities in terms of the lattice-physical parameters removes any explicit dependence upon the lattice spacing in mesonic scattering processes.

Together, these results show that at NLO, the only counterterms entering into the extrapolation formulas for mesonic scattering lengths are the same as the counterterms entering into the physical scattering length at NLO. This lack of unphysical counterterms is desirable from the point of view of chiral extrapolations, but it also has another consequence. Loop graphs in quantum field theories are frequently divergent; there must be a counterterm to absorb these divergences in a consistent field theory. Since there is no counterterm proportional to $a^{2}$ or the sea quark masses, loop graphs involving these quantities are constrained so that they have no divergence proportional to $a^{2}$ or the sea quark masses. This further reduces the pos- 
sible sources of sea quark or lattice spacing dependence. For example, mixed valence-sea meson masses have lattice spacing shifts, so there can be no divergence involving the valence-sea meson masses. In some cases this constraint is strong enough to force the entire valence-sea mass dependence to cancel from scattering lengths expressed in lattice-physical parameters. If this occurs, then the scattering length will not depend on the unknown constant $C_{\text {Mix }}$.

\section{Dependence upon sea quarks}

Now, let us move on to discuss how the NLO extrapolation formulas depend on the particular sea quark discretization in use. At NLO in the effective field theory expansion, mesons composed of one or two sea quarks only arise in loop graphs. In particular, the valence-sea mesons can propagate between vertices where they interact with valence-valence mesons; these interactions involve the LO chiral Lagrangian augmented with the mixing term $a^{2} \mathcal{U}_{V S}$. Because the mixing term is universal, these interaction vertices are the same for all discretization schemes provided LO chiral perturbation theory is applicable. The sea-sea mesons only arise at NLO in hairpins; therefore, they are only sensitive to the quadratic part of the appropriate LO chiral Lagrangian on the sea-sea sector. Thus, we see that our NLO extrapolation formulas only depend on the LO chiral Lagrangian to quadratic order in the sea-sea sector and the LO chiral Lagrangian (with the mixing term) in the valence-sea sector. Together, we see that the condition we require on the sea quark discretization is that the sea-sea sector alone should be described by chiral perturbation theory at $\mathrm{LO}$, and that the constant $C_{\mathrm{Mix}}$ should not be so large that its explicit violation of chiral symmetry overwhelms the dynamical violation of chiral symmetry. Nonlocality which is described by the replica trick does not present a problem since at the level of perturbation theory the analytic continuation required by the replica method is trivial.

Note that the impact of using different sea quark discretizations in our work is only at the level of the quadratic Lagrangian. Therefore, the same NLO extrapolation formulas can be used to describe simulations with different sea quark discretizations, provided that the appropriate mass shifts are taken into account. In the case of staggered sea quarks, the sea-sea mass splitting which occurs in the MA formulas is that of the taste-identity, which has been measured [41], and for the coarse MILC lattices, is given by

$$
a^{2} \Delta_{\text {sea }}=a^{2} \Delta_{I} \simeq(450 \mathrm{MeV})^{2},
$$

for $a \simeq 0.125 \mathrm{fm}$. These mass shifts can only appear through the hairpin interactions at this order. These terms will generally be associated with unphysical MA/PQ effects which give rise to the enhanced chiral logarithms as well as additional finite analytic dependence upon the seasea as well as valence-valence meson masses (and their associated lattice spacing dependent mass corrections). The exception to this is the dependence upon the $\eta$ mass. As can be seen in Eq. (12), the only way the $\eta$ mass dynamically enters processes involving external pions and kaons through $\mathcal{O}\left(\varepsilon_{m}^{2} \varepsilon_{a}^{2}\right)$ is via the mass of the seasea $\eta$, Eq. (13). The other way these discretization effects enter MA formulas is through the mixed valence-sea meson masses, Eq. (10). Currently, this mass shift, $a^{2} \Delta_{\text {Mix }}$, is not known for any type of sea quark discretization. This is one of the more important MA effects, because it enters many quantities of interest at the one-loop level, for both mesons and baryons, and thus to perform chiral extrapolations properly, this mass splitting must be taken into account.

\section{Mixed actions at NNLO}

It is important to note that these conclusions will not hold at NNLO in the effective field theory expansion. At this order, NNLO terms in the effective Lagrangian will introduce $a^{2}$ shifts of the Gasser-Leutwyler parameters themselves. In simulations which are precise enough to be sensitive to NNLO effects in chiral perturbation theory, these effects would have to be removed. In addition, there will be new effects which cannot be absorbed into the Gasser-Leutwyler parameters, but are truly new lattice spacing artifacts. The simplest example to understand is to consider how the pion mass is modified at $\mathcal{O}\left(\varepsilon_{m}^{4} \varepsilon_{a}^{2}\right)$ in a MA theory with staggered sea quarks [70].

Briefly, there will be contributions to the pion mass which break taste, arising, for example, from the GasserLeutwyler operator in Eq. (17) with coefficient $L_{6}$. The taste-breaking contributions to the pion mass arise when the valence pion is contracted with the meson fields in one of the supertraces while the other supertrace is taken over sea-sea mesons which form a loop at this order,

$$
\begin{aligned}
\delta m_{\pi}^{2}(\mathrm{NNLO})= & -\frac{64 m_{\pi}^{2}}{f^{2}} L_{6} N_{s}^{2} \sum_{F, t} n_{t} \frac{B_{0}\left(m_{s_{1}}+m_{s_{2}}\right)}{(4 \pi f)^{2}} \\
& \times \tilde{m}_{s_{1} s_{2}, t}^{2} \ln \left(\frac{\tilde{m}_{s_{1} s_{2}, t}^{2}}{\mu^{2}}\right),
\end{aligned}
$$

where $N_{s}=1 / 4$ is the factor one inserts according to the replica method to account for the 4th root of the sea quark determinant and $n_{t}$ counts the weighting of the mesons of various taste propagating in the loop. The staggered meson mass of flavor $F$, and taste $t$, is given at $\mathrm{LO}$ by $[41,58,71]$

$$
\tilde{m}_{s_{1} s_{2}, t}^{2}=B_{0}\left(m_{s_{1}}+m_{s_{2}}\right)+a^{2} \Delta\left(\xi_{t}\right) .
$$

These taste-breaking effects are unphysical and their associated $\mu$ dependence can only be absorbed by the appropriate unphysical lattice spacing dependent operators arising in the mixed action Lagrangian. This is simply one of many possible examples of how the continuumlike behavior of mixed action theories will break down. 


\section{APPLICATIONS}

In this section, we discuss applications of these results to some specific quantities of physical interest. There have been a number of recent lattice computations [17-25] utilizing the scheme first developed by the LHP Collaboration [72,73] of employing domain wall valence quarks with the publicly available MILC configurations. In particular, the NPLQCD Collaboration has computed the $I=2 \pi \pi$ scattering length [19], ${ }^{17} f_{K} / f_{\pi}$ [23] and determined both the $I=3 / 2$ and $I=1 / 2 K \pi$ scattering lengths through a direct determination of the $I=3 / 2 K \pi$ scattering length [25]. As we will demonstrate by explicit computation, the $I=1 \mathrm{KK}$ scattering length, together with the above three systems, share only two linearly independent sets of counterterms, which are the physical counterterms of interest. Therefore, these four quantities provide a means to test the mixed action formalism with only one lattice spacing. ${ }^{18}$

\section{A. $f_{K} / f_{\pi}$}

The pion and kaon decay constants were computed in a mixed action theory with staggered sea quarks in Ref. [30]. In Appendix $\mathrm{C}$, we include the general form of these results for arbitrary sea quarks to NLO, which we express in terms of the PQ parameters we introduced in Eq. (14). We use these formulas to estimate the error arising from the finite lattice spacing in the recent determination of $f_{K} / f_{\pi}$ in Ref. [23], in which the continuum $\chi \mathrm{PT}$ form of this quantity was used to extrapolate the lattice data to the physical point. The MA functional form of this quantity depends upon the mixed valence-sea meson masses, and so we cannot make a concrete prediction of the error made in this approximation, as the mixed meson mass depends upon $C_{\text {Mix }}$, Eq. (10), which is currently unknown. ${ }^{19}$ Consequently we form the ratio,

\footnotetext{
${ }^{17}$ In addition to the lattice spacing modifications of the $I=2$ $\pi \pi$ scattering length computed in Ref. [34], the exponential finite volume corrections to this quantity were also computed in Ref. [74]. It was found that for the pion masses in use today, these effects were not significant, being on the order of $1 \%$. It is expected that the exponential volume dependence in the other scattering processes will be similar to that of the two-pion system; as in all cases the pion is the lightest particle and will dominate the long range (finite volume) effects.

${ }^{18}$ In Ref. [34], it was argued that to all orders in perturbation theory, the unitarity violating features of MA and PQ theories do not invalidate the known method of extracting infinite volume scattering parameters from finite volume correlation functions [75-77], for all "maximally stretched" two-meson states, i.e. the $I=2 \pi \pi, I=3 / 2 K \pi$, and $I=1 K K$ systems.

${ }^{19}$ In Ref. [35], this quantity was recently estimated by comparing the MA form of the pion form factor to a MA simulation [17]. Unfortunately, only one of the lattice data points was in the chiral regime, so a precise determination of this quantity was not possible.
}

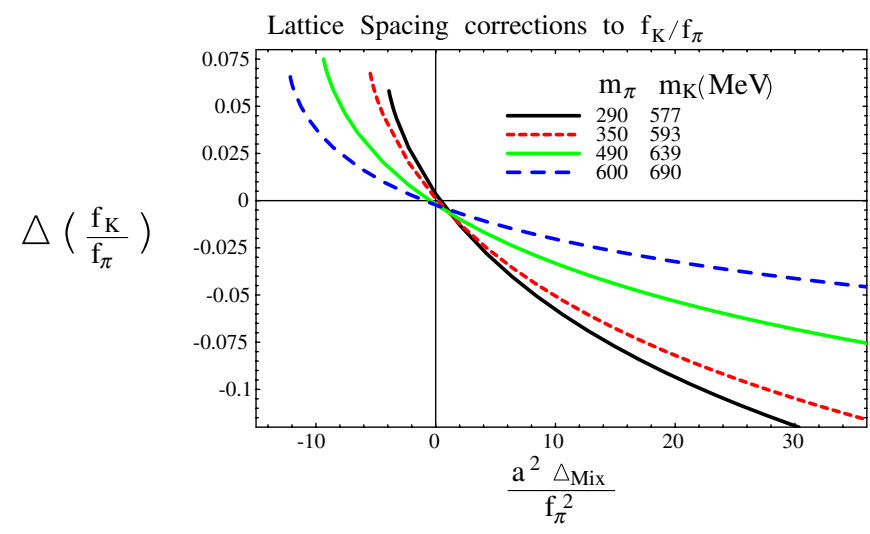

FIG. 1 (color online). We plot the ratio, $\Delta\left(f_{K} / f_{\pi}\right)$ defined in Eq. (28), as a function of the unknown mixed meson mass splitting, $\quad-(600 \mathrm{MeV})^{2} \lesssim a^{2} \Delta_{\text {Mix }} \lesssim(800 \mathrm{MeV})^{2}$. The observed deviation from the continuum $\chi \mathrm{PT}$ formulas is on the order of 5\%, which is important, but not significant enough to directly determine this unknown mass splitting from the MA lattice data of $f_{K} / f_{\pi}$ [23] alone.

$$
\Delta\left(\frac{f_{K}}{f_{\pi}}\right)=\frac{\left.\frac{f_{K}}{f_{\pi}}\right|_{\mathrm{MA}}-\left.\frac{f_{K}}{f_{\pi}}\right|_{\mathrm{QCD}},}{\left.\frac{f_{K}}{f_{\pi}}\right|_{\mathrm{QCD}}},
$$

and in Eq. (C4), we provide the explicit formula for this quantity, with the mass tuning used in Ref. [23] $\left[m_{q_{s}}=\right.$ $\left.m_{q_{v}} \Rightarrow \tilde{\Delta}_{r s}^{2}=\tilde{\Delta}_{j u}^{2}=a^{2} \Delta_{I} \simeq(450 \mathrm{MeV})^{2}\right]$. In Fig. 1, we plot this ratio as a function of the mixed meson splitting in the range $-(600 \mathrm{MeV})^{2} \lesssim a^{2} \Delta_{\text {Mix }} \lesssim(800 \mathrm{MeV})^{2}$. We take the value of $L_{5}(\mu)$ from Ref. [23], as their various fitting procedures produced little variation in the extraction of $L_{5}(\mu)$. This provides us with an indirect means at estimating the error in the extrapolation of the quantity $f_{K} / f_{\pi}$. As can be seen from Fig. 1, reasonable values of $a^{2} \Delta_{\text {Mix }}$ can produce deviations in $f_{K} / f_{\pi}$ on the order of $5 \%$. These deviations are important enough to include in the fitting procedure (although still within the confidence levels in Refs. [23,78]), but not significant enough to determine $a^{2} \Delta_{\text {Mix }}$ directly from the data in Ref. [23]. One also can determine the size of the hairpin contributions alone by setting $a^{2} \Delta_{\text {Mix }}=0$, and, as can be seen in Fig. 1, these effects are a fraction of a percent for all values of the pion mass.

It is important to note that at this order, the counterterm structure of $f_{K} / f_{\pi}$ in a MA theory is identical to the counterterm structure of $f_{K} / f_{\pi}$ in $\chi \mathrm{PT}$, as can be verified by examining Eqs. (C1) and (C2),

$$
\left.\frac{f_{K}}{f_{\pi}}\right|_{\mathrm{MA}} \propto \frac{8\left(m_{K}^{2}-m_{\pi}^{2}\right)}{f^{2}} L_{5}(\mu) .
$$

This can be understood with the arguments presented in Sec. IIB, and the knowledge that the lattice spacing artifacts are flavor blind. 


\section{B. $K K I=1$ scattering length, $a_{K K}^{I=1}$}

Next, we discuss the form of the $I=1 K K$ scattering length, for a MA theory with arbitrary sea quarks, for which the full functional form is provided in Appendix E. The two Kaon system is theoretically ideal for testing the convergence of $S U(3) \chi \mathrm{PT}$, however experimentally much more difficult to study. But recent progress with lattice QCD simulations has allowed the $I=$ $1 K K$ system to be explored within the MA framework. Thus one can use lattice QCD in combination with the appropriate MA effective field theory to explore the convergence of $S U(3) \chi \mathrm{PT}$ [38], or whether a generalized version of $\chi \mathrm{PT}$ is a more appropriate description of nature [79]. In fact it only recently has been confirmed that the standard $S U(2) \chi$ PT power counting is phenomenologically correct [80-82], by comparing our theoretical knowledge of the two-loop $\pi \pi$ scattering [83,84], the pion scalar form factor [85], and the Roy equation analysis [86] with the recent experimental determination of the pion scattering lengths $[87,88]$.

The $I=1 K K$ system has several features in common with the $I=2 \pi \pi$ system discussed in Ref. [34]. First, the $I=1 K K$ system does not have on-shell hairpins in the $s$-channel loops; second, the scattering length does not depend upon the mixed valence-sea mesons when expressed in terms of the lattice-physical parameters; and finally, the only counterterm at NLO is the physical counterterm of interest. The form of the $I=1 K K$ scattering length is given by

$$
\begin{aligned}
m_{K} a_{K K}^{I=1}= & -\frac{m_{K}^{2}}{8 \pi f_{K}^{2}}\left\{1+\frac{m_{K}^{2}}{\left(4 \pi f_{K}\right)^{2}}\left[C_{\pi} \ln \left(\frac{m_{\pi}^{2}}{\mu^{2}}\right)\right.\right. \\
& +C_{K} \ln \left(\frac{m_{K}^{2}}{\mu^{2}}\right)+C_{X} \ln \left(\frac{\tilde{m}_{X}^{2}}{\mu^{2}}\right)+C_{s s} \ln \left(\frac{m_{s s}^{2}}{\mu^{2}}\right) \\
& \left.\left.+C_{0}-32(4 \pi)^{2} L_{K K}^{I=1}(\mu)\right]\right\},
\end{aligned}
$$

where the various coefficients, $C_{\phi}$, are provided in Eqs. (E3)-(E6).

One important point is that the counterterm for the $I=1$ scattering length, $L_{K K}^{I=1}$ is identical to the $I=2 \pi \pi$ scattering length counterterm,

$$
\begin{aligned}
L_{K K}^{I=1} & =L_{\pi \pi}^{I=2} \\
& =2 L_{1}+2 L_{2}+L_{3}-2 L_{4}-L_{5}+2 L_{6}+L_{8} .
\end{aligned}
$$

Before discussing this scattering length in more detail, we first give the result in $\chi \mathrm{PT}$, as this has not been presented in the literature to the authors' knowledge:

$$
\begin{aligned}
m_{K} a_{K K}^{I=1}= & -\frac{m_{K}^{2}}{8 \pi f_{K}^{2}}\left\{1-\frac{m_{K}^{2}}{\left(4 \pi f_{K}\right)^{2}}\left[32(4 \pi) L_{K K}^{I=1}(\mu)\right.\right. \\
& -2 \ln \left(\frac{m_{K}^{2}}{\mu^{2}}\right)+\frac{2 m_{\pi}^{2}}{3\left(m_{\eta}^{2}-m_{\pi}^{2}\right)} \ln \left(\frac{m_{\pi}^{2}}{\mu^{2}}\right) \\
& \left.\left.-\frac{2\left(20 m_{K}^{2}-11 m_{\pi}^{2}\right)}{27\left(m_{\eta}^{2}-m_{\pi}^{2}\right)} \ln \left(\frac{m_{\eta}^{2}}{\mu^{2}}\right)+\frac{14}{9}\right]\right\},
\end{aligned}
$$

with $L_{K K}^{I=1}$ given in Eq. (31), and we have used the leading order meson mass relations to simplify the form of this expression.

The equality of the $I=2 \pi \pi$ and $I=1 K K$ scattering length counterterms allows us to make a prediction for the numerical values of $m_{K} a_{K K}^{I=1}$ one should obtain in a simulation of this system with domain wall valence quarks on the MILC configurations. To do this, we must first convert the counterterm, $l_{\pi \pi}(\mu)$ obtained by NPLQCD in Ref. [19], from the effective theory with two sea flavors to the theory with three sea flavors. For PQ and MA theories, there is an additional subtlety which arises in this matching. If we match the $\chi \mathrm{PT}$ forms of $m_{\pi} a_{\pi \pi}^{I=2}$ in $S U(2)$ to $S U(3)$, then we arrive at the equality (with the conventions defined in Appendix D)

$$
l_{\pi \pi}^{I=2}(\mu)=32(4 \pi)^{2} L_{\pi \pi}^{I=2}(\mu)-\frac{1}{9} \ln \left(\frac{m_{\eta}^{2}}{\mu^{2}}\right)-\frac{1}{9} .
$$

This leads to an exact matching between the $S U(2)$ and $S U(3)$ theories, in which all of the strange quark mass dependence at this order, which is purely logarithmic, is absorbed in the $S U(2)$ Gasser-Leutwyler coefficients [38]. If we naïvely attempt to match the $S U(4 \mid 2)$ to $S U(6 \mid 3)$ MA/PQ expressions for $m_{\pi} a_{\pi \pi}^{I=2}$, using Eqs. (D1) and (D2), one arrives at the relation

$$
\begin{aligned}
l_{\pi \pi}^{I=2}(\mu)= & 32(4 \pi)^{2} L_{\pi \pi}^{I=2}(\mu)-\frac{1}{9} \ln \left(\frac{\tilde{m}_{X}^{2}}{\mu^{2}}\right)-\frac{1}{9} \\
& -\sum_{n=1}^{4}\left(\frac{\tilde{\Delta}_{j u}^{2}}{m_{\pi}^{2}}\right)^{n} \mathcal{F}_{n}\left(m_{\pi}^{2} / \tilde{m}_{X}^{2}\right),
\end{aligned}
$$

where the functions $\mathcal{F}_{n}(y)$ were first determined in Ref. [34] and are given in Eqs. (D3). All of the new terms in this matching arise from the extra hairpin interactions present in the $S U(6 \mid 3)$ theory which are not present in $S U(4 \mid 2)$. One can show that these terms are formally higher order in the $S U(4 \mid 2)$ chiral expansion, but nevertheless we will see that they are not negligible.

The NPLQCD Collaboration recently has computed $m_{\pi} a_{\pi \pi}^{I=2}$ and used the $S U(2)$ extrapolation formula to determine $l_{\pi \pi}^{I=2}$ [19]. Adjusting for conventions and including their largest uncertainty, they determined

$$
l_{\pi \pi}^{I=2}\left(4 \pi f_{\pi}\right) \simeq-10.9 \pm 1.8 .
$$

Starting with this determination, we can then compare the hairpin contributions in $S U(4 \mid 2)$ to those of $S U(6 \mid 3)$ and 
also to the physical contribution at NLO. We collect these results in Table I.

The two-flavor hairpin effects, listed in row (b) of Table I, are not small relative to the (scale independent) $\chi$ PT NLO contributions (a), and for the lightest two masses shown, are of the same order of magnitude. However, when we consider the three flavor theory, we see that the additional hairpin effects (c) are of the same order as the twoflavor hairpin contributions (which also contribute in the three flavor theory), but opposite in sign. Taking into account all of the hairpin contributions by using the three flavor theory, one observes that the sum of these unphysical effects (d) is approximately an order of magnitude smaller than the physical NLO effects of $\chi \mathrm{PT}$, but increases in importance as the pion mass is reduced. This justifies our assumption of the determination of $l_{\pi \pi}^{I=2}$ given in Eq. (35). This also justifies the $S U(3) \rightarrow S U(2)$ matching given in Eq. (33) and explains the success found in Ref. [19] of using the $S U(2) \chi \mathrm{PT}$ formula to determine $l_{\pi \pi}^{I=2}$, as the unphysical hairpin corrections to this formula provide a relative shift of about $10 \%$ to the NLO contributions for the masses simulated, which is roughly the size of their largest quoted error.

In Fig. 2, we plot the absolute values of the various NLO contributions to the $I=2 \pi \pi$ scattering length as a function of the pion mass, which highlight the importance of these hairpin effects. Their relative importance is enhanced for the values listed in Table I because of the large cancellation of the counterterm and chiral log at NLO (a). It is clear that these effects will become more important as one moves further into the chiral regime $\left(m_{\pi} \rightarrow 0\right)$.

Given the small contribution of the NLO hairpin effects to $m_{\pi} a_{\pi \pi}^{I=2}$, we can use the determination of $l_{\pi \pi}^{I=2}(\mu)$ in Eq. (35) [19], and the matching of Eq. (33) to determine $L_{K K}^{I=1}(\mu)$ and thus predict values of $m_{K} a_{K K}^{I=1}$, which we provide in Table II. We provide both the comparison of the NLO effects as predicted by both the MA theory as well as $S U(3) \chi \mathrm{PT}$, which we compare to the tree level prediction, as well as the total scattering length through NLO. We find that similar to $m_{\pi} a_{\pi \pi}^{I=2}$, the NLO hairpin effects for $m_{K} a_{K K}^{I=1}$ are only about $10 \%$ of the NLO $\chi \mathrm{PT}$ value, less than the accuracy we claim here. We find that a current MA

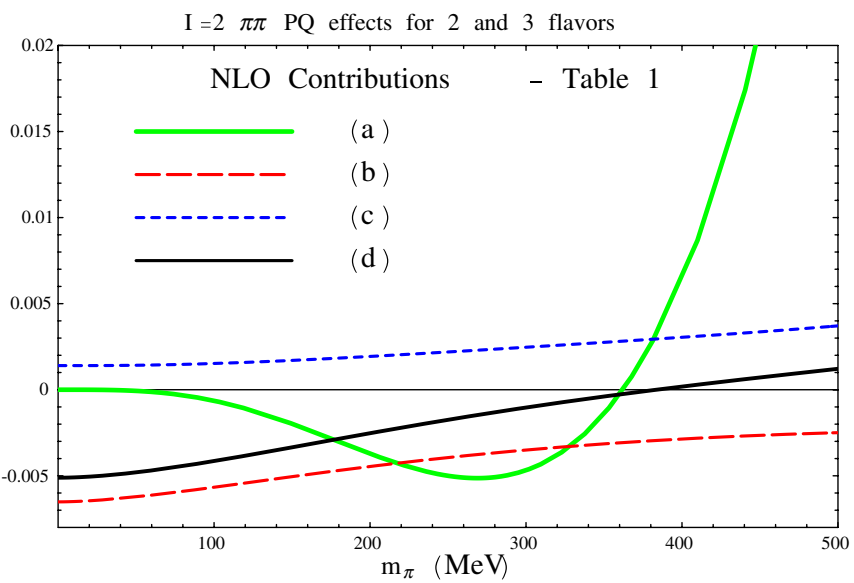

FIG. 2 (color online). We plot the absolute values of the various NLO contributions to $m_{\pi} a_{\pi \pi}^{I=2}$ listed in Table I. The NLO $\chi$ PT contribution is given by (a), which demonstrates the large cancellation of the counterterm and the chiral log for light to medium pion masses. The long dashed bottom curve is the two-sea flavor hairpin effects (b), which are the same order of magnitude as (a), for $m_{\pi} \lesssim 400 \mathrm{MeV}$. When the new three-sea flavor hairpin effects (c) are added to the two-sea flavor effects, one finds that the total three-sea flavor hairpin effects (d) (solid curve) are small compared to (a) for $m_{\pi} \gtrsim 250 \mathrm{MeV}$.

lattice determination of $m_{K} a_{K K}^{I=1}$ will not be sensitive to the unphysical hairpin contributions with the expected level of uncertainty, as can be seen by the predicted MA and $S U(3)$ values. However, for both the MA and $S U(3)$ theories, the NLO contributions are 15-30\% correction to the LO term showing a convergence expected by power counting.

The first error is due to the uncertainty in the determination of $L_{K K}^{I=1}$ we obtain from the matching in Eq. (34) and the extraction of $l_{\pi \pi}^{I=2}$ from Ref. [19], Eq. (35). This uncertainty includes estimations of the two-loop contributions to $m_{\pi} a_{\pi \pi}^{I=2}$ in $S U(2) \chi \mathrm{PT}$. The second uncertainty listed in Table II is a power counting estimation of the NNLO contributions to $m_{K} a_{K K}^{I=1}$. Some of these effects are already included in the first uncertainty but a conservative estimate of our predicted error is to add these uncertainties in quadrature.

TABLE I. Hairpin contributions to $m_{\pi} a_{\pi \pi}^{I=2}$. We provide the various hairpin contributions to the $I=2 \pi \pi$ scattering length for both the two-sea flavor (b) and three-sea flavor theory (d), which we compare to the $\chi$ PT NLO contribution (a) and LO contribution, top row. In row (c), we give the new hairpin effects which arise in the 3-flavor theory, and in (d) we provide the total three-sea flavor hairpin effects.

\begin{tabular}{|c|c|c|c|c|c|}
\hline & $m_{\pi}(\mathrm{MeV})$ & 293 & 354 & 493 & 592 \\
\hline & $-\frac{m_{\pi}^{2}}{8 \pi f_{\pi}^{2}}$ & -0.156 & -0.218 & -0.372 & -0.483 \\
\hline (a) & $-\frac{2 \pi m_{\pi}^{4}}{\left(4 \pi f_{\pi}\right)^{4}}\left[3 \ln \left(\frac{m_{\pi}^{2}}{\mu^{2}}\right)-1-l_{\pi \pi}^{I=2}(\mu)\right]$ & 0.00460 & 0.00140 & -0.0314 & -0.0818 \\
\hline (b) & $-\frac{2 \pi m_{\pi}^{4}}{\left(4 \pi f_{\pi}\right)^{4}}\left[-\frac{\tilde{\Delta}_{j u}^{4}}{6 m_{\pi}^{4}}\right]$ & 0.00359 & 0.00327 & 0.00254 & 0.00207 \\
\hline (c) & $-\frac{2 \pi m_{\pi}^{4}}{\left(4 \pi f_{\pi}\right)^{4}}\left[\sum_{\tilde{\tau}=1}^{4}\left(\frac{\tilde{\Delta}_{j u}}{m_{\pi}^{2}}\right)^{n} \mathcal{F}_{n}\left(m_{\pi}^{2} / \tilde{m}_{X}^{2}\right)\right]$ & -0.00243 & -0.00289 & -0.00371 & -0.00396 \\
\hline (d) & $-\frac{2 \pi m_{\pi}^{4}}{\left(4 \pi f_{\pi}^{4}\right)^{4}}\left[-\frac{\tilde{\Delta}_{j u}^{4}}{6 m_{\pi}^{4}}+\sum_{n=1}^{4}\left(\frac{\tilde{\Delta}_{j u}}{m_{\pi}^{2}}\right)^{n} \mathcal{F}_{n}\left(m_{\pi}^{2} / \tilde{m}_{X}^{2}\right)\right]$ & 0.00116 & 0.00040 & -0.00117 & -0.00188 \\
\hline
\end{tabular}


TABLE II. Predictions of $m_{K} a_{K K}^{I=1}$. We use the equality of the $m_{K} a_{K K}^{I=1}$ and $m_{\pi} a_{\pi \pi}^{I=2}$ counterterms (expressed in lattice-physical parameters) to predict the values of $m_{K} a_{K K}^{I=1}$ which would be computed in a MA lattice formulation with domain wall valence quarks on the MILC staggered sea quarks, which we compare both to the tree level prediction as well as the $S U(3) \chi \mathrm{PT}$ prediction, for values of $m_{K} / f_{K}$ taken from Refs. [19,23]. We also provide a prediction of the scattering length at the physical point. The first error is due to the uncertainty in the determination of $L_{K K}^{I=1}$ from Eq. (34) and the value of $l_{\pi \pi}^{I=2}$, Eq. (35) determined in Ref. [19]. The second error is a power counting estimate of the NNLO contributions to the scattering length.

\begin{tabular}{l|cccc}
\hline \hline$m_{K}: f_{K}(\mathrm{MeV})$ & $577: 172$ & $593: 171$ & $639: 173$ & $690: 177$ \\
\hline$m_{K} a_{K K}^{I=1}(\mathrm{LO}):-\frac{m_{K}^{2}}{8 \pi f_{K}^{2}}$ & -0.447 & -0.479 & -0.542 & -0.605 \\
$m_{K} a_{K K}^{I=1}(\mathrm{NLO}: \mathrm{MA})$ & -0.091 & -0.113 & -0.162 & -0.223 \\
$m_{K} a_{K K}^{I=1}(\mathrm{NLO}: S U(3))$ & -0.084 & -0.107 & -0.157 & -0.217 \\
\hline$m_{K} a_{K K}^{I=1}(\mathrm{MA})$ & $-0.540 \pm 0.069 \pm 0.026$ & $-0.592 \pm 0.079 \pm 0.031$ & $-0.704 \pm 0.102 \pm 0.048$ & $-0.828 \pm 0.127 \pm 0.072$ \\
$m_{K} a_{K K}^{I=1}(S U(3))$ & $-0.531 \pm 0.069 \pm 0.026$ & $-0.586 \pm 0.079 \pm 0.031$ & $-0.699 \pm 0.102 \pm 0.048$ & $-0.823 \pm 0.127 \pm 0.072$ \\
\hline physical point & $496: 161$ & & & \\
$m_{K} a_{K K}^{I=1}(S U(3))$ & $-0.424 \pm 0.049 \pm 0.012$ & & & \\
\hline \hline
\end{tabular}

\section{C. $K \pi I=3 / 2$ scattering length, $a_{K \pi}^{I=3 / 2}$}

The $K \pi$ system is also an interesting laboratory for exploring the three flavor structure of low-energy hadron interactions, and moreover it is experimentally accessible with proposed studies by the DIRAC Collaboration [89]. There recently has been a direct MA lattice QCD determination of the $I=3 / 2 K \pi$ scattering length, which in combination with the theoretical knowledge of the NLO $\chi \mathrm{PT} I=1 / 2$ and $I=3 / 2 K \pi$ scattering lengths [90-93] has allowed a determination of both isospin scattering lengths [25]. There is additionally a two-loop computation of $K \pi$ scattering in $S U(3) \chi \mathrm{PT}$ which studies the convergence of the theory with standard power counting [94]. Before embarking on a study of the two-loop effects with lattice QCD, one must first understand the lattice corrections at NLO. This is the motivation for this section.

The tree level $I=3 / 2 K \pi$ scattering length is given by

$$
\begin{aligned}
\left(m_{\pi}+m_{K}\right) a_{K \pi}^{I=3 / 2} & =-\frac{m_{\pi} m_{K}}{4 \pi f_{K} f_{\pi}}, \quad \text { or } \\
\mu_{K \pi} a_{K \pi}^{I=3 / 2} & =-\frac{\mu_{K \pi}^{2}}{4 \pi f_{K} f_{\pi}},
\end{aligned}
$$

where $\mu_{K \pi}$ is the reduced mass of the $K \pi$ system. We chose to express our extrapolation formulas in terms of the product $f_{K} f_{\pi}$ since this symmetric treatment of the $K$ and $\pi$ mesons provides the simplest form of the scattering length. We find, however, that the $I=\frac{3}{2}$ scattering length still depends on the mixed valence-sea meson masses, and therefore on the parameter $C_{\mathrm{Mix}}$. Consequently, accurate chiral extrapolations of this scattering length will require a determination of the value of $C_{\mathrm{Mix}}$ appropriate to the particular sea quark discretization used in the simulation. The form of the MA $I=3 / 2 K \pi$ scattering length is

$$
\begin{aligned}
\mu_{K \pi} a_{K \pi}^{I=3 / 2}= & -\frac{\mu_{K \pi}^{2}}{4 \pi f_{K} f_{\pi}}\left[1-\frac{32 m_{K} m_{\pi}}{f_{K} f_{\pi}} L_{\pi \pi}^{I=2}(\mu)\right. \\
& \left.+\frac{8\left(m_{K}-m_{\pi}\right)^{2}}{f_{K} f_{\pi}} L_{5}(\mu)\right] \\
& +\mu_{K \pi}\left[a_{v v}^{K \pi, 3 / 2}(\mu)+a_{v s}^{K \pi, 3 / 2}(\mu)\right],
\end{aligned}
$$

where $a_{v v}^{K \pi, 3 / 2}(\mu)$ is the valence-valence (including valence-ghost) contribution to the scattering length and $a_{v s}^{K \pi, 3 / 2}(\mu)$ is a nonvanishing contribution from mixed valence-sea mesons to the scattering length. The other important thing to note is that there are two counterterms for this scattering length which can both be determined through its chiral extrapolation formula, but can also independently be determined in other processes; $L_{5}(\mu)$ can be determined independently by $f_{K} / f_{\pi}$ and $L_{\pi \pi}^{I=3 / 2}(\mu)$ can be determined either with $I=2 \pi \pi$ or $I=1 K K$ scattering. Now we have explicitly demonstrated that the four observable quantities, $a_{\pi \pi}^{I=2}, a_{K K}^{I=1}, a_{K \pi}^{I=3 / 2}$, and $f_{K} / f_{\pi}$, when expressed in terms of the lattice-physical parameters, only share two linearly independent counterterms through NLO in MA (and PQ) $\chi$ PT.

Before continuing, we provide the continuum $S U(3)$ $\chi \mathrm{PT}$ form of $a_{K \pi}^{I=3 / 2}$, which is the same as can be constructed from Ref. [93] with the NLO shift of $f_{K} \rightarrow f_{\pi}$,

$$
\begin{aligned}
\mu_{K \pi} a_{K \pi}^{I=3 / 2}= & -\frac{\mu_{K \pi}^{2}}{4 \pi f_{K} f_{\pi}}\left\{1+\frac{1}{(4 \pi)^{2} f_{K} f_{\pi}}\left[\kappa_{\pi} \ln \left(\frac{m_{\pi}^{2}}{\mu^{2}}\right)+\kappa_{K} \ln \left(\frac{m_{K}^{2}}{\mu^{2}}\right)+\kappa_{\eta} \ln \left(\frac{m_{\eta}^{2}}{\mu^{2}}\right)-\frac{86}{9} m_{K} m_{\pi}\right.\right. \\
& \left.\left.+\kappa_{\tan } \arctan \left(\frac{2\left(m_{K}-m_{\pi}\right) \sqrt{2 m_{K}^{2}+m_{K} m_{\pi}-m_{\pi}^{2}}}{\left(2 m_{K}-m_{\pi}\right)\left(m_{K}+2 m_{\pi}\right)}\right)\right]-\frac{32 m_{K} m_{\pi}}{f_{K} f_{\pi}} L_{\pi \pi}^{I=2}(\mu)+\frac{8\left(m_{K}-m_{\pi}\right)^{2}}{f_{K} f_{\pi}} L_{5}(\mu)\right\}
\end{aligned}
$$


with

$$
\begin{gathered}
\kappa_{\pi}=-\frac{m_{\pi}^{2}}{4} \frac{11 m_{K}^{2}+22 m_{K} m_{\pi}-5 m_{\pi}^{2}}{m_{K}^{2}-m_{\pi}^{2}} \\
\kappa_{K}=\frac{m_{K}}{18} \frac{134 m_{K}^{2} m_{\pi}-9 m_{K}^{3}+55 m_{K} m_{\pi}^{2}-16 m_{\pi}^{3}}{m_{K}^{2}-m_{\pi}^{2}} \\
\kappa_{\eta}=\frac{-36 m_{K}^{3}-12 m_{K}^{2} m_{\pi}+m_{K} m_{\pi}^{2}+9 m_{\pi}^{3}}{36\left(m_{K}-m_{\pi}\right)} \\
\kappa_{\tan }=\frac{16 m_{K} m_{\pi}}{9} \frac{\sqrt{2 m_{K}^{2}+m_{K} m_{\pi}-m_{\pi}^{2}}}{m_{K}-m_{\pi}}
\end{gathered}
$$

In Appendix F, we provide the full form of the MA $I=$ $3 / 2 K \pi$ scattering length. Here we wish to examine the valence-sea contribution in more detail. This contribution to the scattering length is given by ${ }^{20}$

$$
\begin{aligned}
\mu_{K \pi} a_{v s}^{K \pi, 3 / 2}(\mu)= & -\frac{\mu_{K \pi}^{2}}{4 \pi f_{K} f_{\pi}} \frac{1}{2(4 \pi)^{2} f_{K} f_{\pi}} \sum_{F=j, l, r} \\
& \times\left[C_{F s} \ln \left(\frac{\tilde{m}_{F s}^{2}}{\mu^{2}}\right)-C_{F d} \ln \left(\frac{\tilde{m}_{F d}^{2}}{\mu^{2}}\right)\right. \\
& \left.+4 m_{K} m_{\pi} J\left(\tilde{m}_{F d}^{2}\right)\right],
\end{aligned}
$$

where

$$
\begin{gathered}
C_{F s}=\frac{4 m_{K}^{2} m_{\pi}-\tilde{m}_{F s}^{2}\left(m_{K}+m_{\pi}\right)}{m_{K}-m_{\pi}}, \\
C_{F d}=\frac{4 m_{K} m_{\pi}^{2}-\tilde{m}_{F d}^{2}\left(m_{K}+m_{\pi}\right)}{m_{K}-m_{\pi}}, \\
J(M)=2 \frac{\sqrt{M^{2}-m_{\pi}^{2}}}{m_{K}-m_{\pi}} \arctan \left(\frac{\left(m_{K}-m_{\pi}\right) \sqrt{M^{2}-m_{\pi}^{2}}}{M^{2}+m_{K} m_{\pi}-m_{\pi}^{2}}\right) \\
-m_{K} m_{\pi} .
\end{gathered}
$$

The scale dependence in $a_{v s}^{K \pi, 3 / 2}(\mu)$ can be shown to be

$$
a_{v s}^{K \pi, 3 / 2}(\mu) \propto \sum_{F=j, l, r}-\ln \left(\mu^{2}\right)\left(m_{K}-m_{\pi}\right)^{2},
$$

and as claimed, independent of both the lattice spacing $a$ and the sea quark masses, and is absorbed by $L_{5}(\mu)$. We stress again that the $I=3 / 2 K \pi$ scattering length depends

\footnotetext{
${ }^{20}$ We note that the summation over sea flavor $F$ implicitly includes the appropriate factors for staggered sea quarks, the sum over taste and the factors of $N_{s}=1 / 4$ which arise from the 4th-rooting trick. For other brands of quarks, this is simply a sum over the sea flavors, $j, l$, and $r$.
}

upon mixed valence-sea mesons, which receive lattice spacing dependent mass shifts proportional to the unknown quantity $C_{\mathrm{Mix}}$. This quantity is currently unknown for all variants of MA lattice QCD and must be determined for a correct extrapolation of MA lattice QCD simulations. For this reason, we do not provide a table with postdictions of $\mu_{K \pi} a_{K \pi}^{I=3 / 2}$.

\section{DISCUSSION}

Mixed action simulations provide a promising solution to the problem of performing fully dynamical simulations with light quarks which are under theoretical control. Recently, several simulations have been performed using the publicly available MILC staggered lattices with domain wall valence quarks, and the future for simulations using Wilson sea quarks and GW valence quarks seems to be bright. This work shows that mixed action simulations with Ginsparg-Wilson valence quarks are theoretically clean. We have shown that the counterterms appearing in mesonic scattering lengths are precisely those that occur in QCD, so that one can in principle measure these counterterms with a single lattice spacing. We also find that the same chiral extrapolation formulas can be used to describe mixed action simulations with GW quarks with mild restrictions on the type of sea quark discretization usedprovided, of course, that QCD is recovered in the continuum limit. Thus, our results hold for simulations with domain wall and overlap quarks, Wilson quarks $[\mathcal{O}(a)$ improved and twisted mass quarks at maximal twist] as well as simulations using rooted staggered quarks (assuming that the 4th-rooting procedure is valid and that the replica method correctly captures all of the nonlocality introduced by the rooting procedure-which has been argued to all orders in perturbation theory [95]).

It was previously observed in Ref. [34] that the $\pi \pi$ scattering length does not depend on the parameter $C_{\text {Mix }}$ of mixed action chiral perturbation theory. Here, we find that this also holds for the $K K$ scattering length. However, the $K \pi$ scattering length does depend on $C_{\mathrm{Mix}}$, and, therefore, accurate chiral extrapolations of mixed action data will require a measurement of this quantity. However, we also have computed the ratio $f_{K} / f_{\pi}$ in mixed action chiral perturbation theory, which depends upon $C_{\text {Mix }}$. By varying $C_{\text {Mix }}$ over a broad range of values, we find the impact to be modest, on the order of 5\%. In addition, taking into account the small hairpin corrections to $m_{\pi} a_{\pi \pi}^{I=2}$ [34] and the equally small predicted corrections to $m_{K} a_{K K}^{I=1}$, Table II, we expect the impact of $C_{\mathrm{Mix}}$ on $a_{K \pi}^{I=3 / 2}$ to also be small at this order of precision, $\mathcal{O}\left(\varepsilon_{m}^{4}, \varepsilon_{m}^{2} \varepsilon_{a}^{2}, \varepsilon_{a}^{4}\right)$. In Table II we provide predictions of $m_{K} a_{K K}^{I=1}$ for various values of $m_{K}$ and also provide a prediction at the physical point.

In Sec. IIB, we have demonstrated why the use of latticephysical parameters (or on-shell renormalization) significantly simplifies the form of the extrapolation formulas for mesonic systems. We stress that these arguments do not 
depend upon the momentum of the system, nor upon having only two external mesons, and thus will be applicable not just for scattering lengths, but also for other scattering parameters, such as the effective range, as well as for $N>$ 2 mesonic systems. In the appendices we have provided explicit NLO extrapolation formulas for the meson masses and decay constants as well as the three scattering lengths discussed in this paper, for arbitrary sea quark discretization schemes, expressed in terms of the PQ parameters we introduced in Eq. (14). A thorough understanding of the lattice spacing effects at this order will require knowledge of the counterterms in the masses and decay constants.

We would like to conclude with a small point and a few suggestions. If one is interested in removing the unitarity violating effects in MA lattice simulations, for the lowenergy dynamics of the system, then theoretical analysis unambiguously advocates the tuning $\tilde{\Delta}_{r s}=\tilde{\Delta}_{j u}=0$, which is the generalization of $m_{q_{\text {sea }}}-m_{q_{\mathrm{val}}}=0$ for PQ theories. This is the most QCD-like scenario for MA theories in which the unitarity violating double pole propagators in Eq. (12) are tuned to zero. It has been shown recently that this double pole structure of the flavor-neutral propagators persists to all orders in $\mathrm{PQ} \chi \mathrm{PT}$ [96], and thus this will be the appropriate tuning to higher orders as well. From the point of view of doing chiral physics, this is not desirable for the coarse MILC lattices, as the lattice spacing shift to the taste-identity staggered mesons is $a^{2} \Delta_{I} \simeq$ $(450 \mathrm{MeV})^{2}$, which would make for heavy pions. Therefore, we caution users of MA lattice simulations to remember the existence of these unitarity violating effects present in current MA simulations.

The simplified form of MA/PQ extrapolation formulas for the two-meson systems is particularly dependent upon the implications of the chiral symmetry of the valence quarks. However, we conjecture that a similar, but not as strong, simplification will occur for other hadronic observables, in particular, for nuclear physics as well as heavy meson observables, if also expressed in terms of latticephysical parameters, which will lead to improved chiral extrapolations. This is supported by the recent fits of the NPLQCD Collaboration [19-23,25] and the LHP Collaboration [97]. Based upon our theoretical understanding of effective field theories designed to incorporate lattice spacing artifacts, we expect that even for fermion discretization schemes which do not have chiral symmetry, the use of lattice-physical parameters (on-shell renormalization) will in general simplify the chiral extrapolation formulas and improve chiral fits.

\section{ACKNOWLEDGMENTS}

We would like to thank Martin Savage for many useful discussions. We also thank Ruth Van de Water for her input at the start of this project. J.W.C. is supported by the National Science Council of R.O.C. D. O. C. is supported in part by the U.S. DOE under the Grant No. DE-FG039ER40701. A. W.L. was supported under DOE Grants No. DE-FG03-97ER-41014 and No. DE-FG02-93ER40762.

\section{APPENDIX A: $m_{\pi}$ AND $f_{\pi}$ FOR 2 SEA FLAVORS}

In this appendix, we provide the explicit formulas for the pion mass and decay constant in a two-sea flavor MA theory. These were first computed in Refs. $[15,30]$. Here we provide the answers expressed in terms of the PQ parameters we introduced in Eq. (14).

$$
\begin{aligned}
m_{\pi}^{2}= & 2 B_{0} \hat{m}\left\{1+\frac{m_{\pi}^{2}}{(4 \pi f)^{2}} \ln \left(\frac{m_{\pi}^{2}}{\mu^{2}}\right)-\frac{m_{\pi}^{2}}{f^{2}} \ell^{(m)}(\mu)\right. \\
- & \frac{\tilde{\Delta}_{j u}^{2}}{(4 \pi f)^{2}}\left[1+\ln \left(\frac{m_{\pi}^{2}}{\mu^{2}}\right)\right]-\frac{\Delta_{j u}^{2}}{f^{2}} \ell_{\mathrm{PQ}}^{(m)}(\mu) \\
+ & \left.\frac{a^{2}}{f^{2}} \ell_{a^{2}}^{(m)}(\mu)\right\} . \\
f_{\pi}= & f\left\{1-\frac{2 \tilde{m}_{j u}^{2}}{(4 \pi f)^{2}} \ln \left(\frac{\tilde{m}_{j u}^{2}}{\mu^{2}}\right)+\frac{m_{\pi}^{2}}{f^{2}} \ell^{(f)}(\mu)\right. \\
& \left.+\frac{\Delta_{j u}^{2}}{f^{2}} \ell_{\mathrm{PQ}}^{(f)}(\mu)+\frac{a^{2}}{f^{2}} \ell_{a^{2}}^{(f)}(\mu)\right\} .
\end{aligned}
$$

\section{APPENDIX B: MESON MASSES}

In this appendix we collect the pion and kaon mass and decay constant for a three-sea flavor MA theory. These were first computed in Refs. [15,30]. Here we provide the answers expressed in terms of the PQ parameters we introduced in Eq. (14).

$$
\begin{aligned}
m_{\pi}^{2}= & 2 B_{0} \hat{m}\left\{1+\ln \left(\frac{m_{\pi}^{2}}{\mu^{2}}\right)\left[\frac{m_{\pi}^{2}}{(4 \pi f)^{2}}-\frac{\tilde{\Delta}_{j u}^{2}\left(3 \tilde{m}_{X}^{2}-m_{\pi}^{2}\right)}{3(4 \pi f)^{2}\left(\tilde{m}_{X}^{2}-m_{\pi}^{2}\right)}+\frac{\tilde{\Delta}_{j u}^{4} \tilde{m}_{X}^{2}}{3(4 \pi f)^{2}\left(\tilde{m}_{X}^{2}-m_{\pi}^{2}\right)^{2}}\right]+\frac{\tilde{\Delta}_{j u}^{4}}{3(4 \pi f)^{2}\left(\tilde{m}_{X}^{2}-m_{\pi}^{2}\right)}\right. \\
& -\ln \left(\frac{\tilde{m}_{X}^{2}}{\mu^{2}}\right)\left[\frac{\tilde{m}_{X}^{2}}{3(4 \pi f)^{2}}-\frac{\tilde{\Delta}_{j u}^{2} \tilde{m}_{X}^{2}}{3(4 \pi f)^{2}\left(\tilde{m}_{X}^{2}-m_{\pi}^{2}\right)}\left(2-\frac{\tilde{\Delta}_{j u}^{2}}{\left(\tilde{m}_{X}^{2}-m_{\pi}^{2}\right)}\right)\right]-\left(\frac{32 \Delta_{j u}^{2}}{f^{2}}+\frac{16 \Delta_{r s}^{2}}{f^{2}}\right)\left[L_{4}(\mu)-2 L_{6}(\mu)\right] \\
& \left.-\frac{16 m_{\pi}^{2}}{f^{2}}\left[L_{4}(\mu)+L_{5}(\mu)-2 L_{6}(\mu)-2 L_{8}(\mu)\right]-\frac{32 m_{K}^{2}}{f^{2}}\left[L_{4}(\mu)-2 L_{6}(\mu)\right]-\frac{\tilde{\Delta}_{j u}^{2}}{(4 \pi f)^{2}}+\frac{a^{2}}{f^{2}} L_{m a^{2}}(\mu)\right\} .
\end{aligned}
$$




$$
\begin{aligned}
m_{K}^{2}= & B_{0}\left(\hat{m}+m_{s}\right)-\frac{16 m_{K}^{4}}{f^{2}}\left[2 L_{4}(\mu)+L_{5}(\mu)-4 L_{6}(\mu)-2 L_{8}(\mu)\right]-\frac{16 m_{K}^{2} m_{\pi}^{2}}{f^{2}}\left[L_{4}(\mu)-2 L_{6}(\mu)\right] \\
& -\frac{16 m_{K}^{2}}{f^{2}}\left(2 \Delta_{j u}^{2}+\Delta_{r s}^{2}\right)\left[L_{4}(\mu)-2 L_{6}(\mu)\right]+\frac{a^{2} m_{K}^{2}}{f^{2}} L_{m a^{2}}(\mu)+\ln \left(\frac{\tilde{m}_{X}^{2}}{\mu^{2}}\right)\left[\frac{2 m_{K}^{2} \tilde{m}_{X}^{2}}{3(4 \pi f)^{2}}-\frac{\tilde{\Delta}_{j u}^{2} \tilde{m}_{X}^{2}\left(8 m_{K}^{2}+3 \tilde{m}_{X}^{2}+m_{\pi}^{2}\right)}{18(4 \pi f)^{2}\left(\tilde{m}_{X}^{2}-m_{\pi}^{2}\right)}\right. \\
& \left.+\frac{2 \tilde{\Delta}_{r s}^{2} \tilde{m}_{X}^{2} m_{K}^{2}}{18(4 \pi f)^{2}\left(\tilde{m}_{X}^{2}-m_{\pi}^{2}\right)}-\frac{\tilde{\Delta}_{j u}^{2} \tilde{\Delta}_{r s}^{2} \tilde{m}_{X}^{2}\left(\tilde{m}_{X}^{2}+4 m_{K}^{2}+m_{\pi}^{2}\right)}{3(4 \pi f)^{2}\left(\tilde{m}_{X}^{2}+m_{\pi}^{2}-2 m_{K}^{2}\right)}+\frac{\tilde{\Delta}_{j u}^{2}}{9(4 \pi f)^{2}\left(\tilde{m}_{X}^{2}-m_{\pi}^{2}\right)\left(\tilde{m}_{X}^{2}+m_{\pi}^{2}-2 m_{K}^{2}\right)}\right] \\
& +\ln \left(\frac{m_{\pi}^{2}}{\mu^{2}}\right) \frac{\tilde{\Delta}_{j u}^{2} m_{\pi}^{2}}{(4 \pi f)^{2}}\left[\frac{3 \tilde{m}_{X}^{2}+8 m_{K}^{2}+m_{\pi}^{2}}{18\left(\tilde{m}_{X}^{2}-m_{\pi}^{2}\right)}-\frac{\left.\tilde{\Delta}_{K}^{2}+m_{\pi}^{2}\right)}{18\left(\tilde{m}_{X}^{2}-m_{\pi}^{2}\right)}+\frac{\tilde{\Delta}_{j u}^{2}\left(2 m_{K}^{2}-m_{\pi}^{2}\right)}{9\left(m_{\pi}^{2}-m_{\pi}^{2}\right)\left(\tilde{m}_{X}^{2}-m_{\pi}^{2}\right)}\right] \\
& +\ln \left(\frac{m_{s s}^{2}}{\mu^{2}}\right) \frac{\tilde{\Delta}_{r s}^{2} m_{K}^{2}}{(4 \pi f)^{2}}\left[\frac{2\left(2 m_{K}^{2}-m_{\pi}^{2}\right)}{3\left(\tilde{m}_{X}^{2}+m_{\pi}^{2}-2 m_{K}^{2}\right)}-\frac{\tilde{m}_{K}^{2}}{3\left(m_{K}^{2}-m_{\pi}^{2}\right)\left(\tilde{m}_{X}^{2}+m_{\pi}^{2}-2 m_{K}^{2}\right)}\right] .
\end{aligned}
$$

Note that the lattice spacing dependent counterterms for the meson masses have the same coefficient. This is because the discretization scheme is flavor blind.

\section{APPENDIX C: DECAY CONSTANTS AND $f_{K} / f_{\pi}$}

The pion decay constant is given by

$$
\begin{aligned}
f_{\pi}= & f\left\{1-\frac{2 \tilde{m}_{j u}^{2}}{(4 \pi f)^{2}} \ln \left(\frac{\tilde{m}_{j u}^{2}}{\mu^{2}}\right)-\frac{\tilde{m}_{r u}^{2}}{(4 \pi f)^{2}} \ln \left(\frac{\tilde{m}_{r u}^{2}}{\mu^{2}}\right)+\frac{8 m_{\pi}^{2}}{f^{2}}\left[L_{5}(\mu)+L_{4}(\mu)\right]+\frac{16 m_{K}^{2}}{f^{2}} L_{4}(\mu)+\frac{8\left(2 \Delta_{j u}^{2}+\Delta_{r s}^{2}\right)}{f^{2}} L_{4}(\mu)\right. \\
& \left.+\frac{a^{2}}{f^{2}} L_{f a^{2}}(\mu)\right\}
\end{aligned}
$$

while the kaon decay constant is

$$
\begin{aligned}
f_{K}= & f\left\{1-\frac{\tilde{m}_{s j}^{2}}{(4 \pi f)^{2}} \ln \left(\frac{\tilde{m}_{s j}^{2}}{\mu^{2}}\right)-\frac{\tilde{m}_{r u}^{2}}{2(4 \pi f)^{2}} \ln \left(\frac{\tilde{m}_{r u}^{2}}{\mu^{2}}\right)-\frac{\tilde{m}_{j u}^{2}}{(4 \pi f)^{2}} \ln \left(\frac{\tilde{m}_{j u}^{2}}{\mu^{2}}\right)-\frac{\tilde{m}_{r s}^{2}}{2(4 \pi f)^{2}} \ln \left(\frac{\tilde{m}_{r s}^{2}}{\mu^{2}}\right)+\frac{8 m_{K}^{2}}{f^{2}}\left(L_{5}(\mu)+2 L_{4}(\mu)\right)\right. \\
& +\frac{8 m_{\pi}^{2}}{f^{2}} L_{4}(\mu)+\frac{8\left(2 \Delta_{j u}^{2}+\Delta_{r s}^{2}\right)}{f^{2}} L_{4}(\mu)+\frac{a^{2}}{f^{2}} L_{f a^{2}}(\mu)-\frac{\tilde{\Delta}_{j u}^{2}}{4(4 \pi f)^{2}}+\frac{\tilde{\Delta}_{j u}^{4}}{12(4 \pi f)^{2}\left(\tilde{m}_{X}^{2}-m_{\pi}^{2}\right)}+\frac{\tilde{\Delta}_{r s}^{2}\left(m_{K}^{2}-m_{\pi}^{2}\right)}{3(4 \pi f)^{2}\left(\tilde{m}_{X}^{2}-m_{s s}^{2}\right)} \\
& -\frac{\tilde{\Delta}_{j u}^{2} \tilde{\Delta}_{r s}^{2}}{6(4 \pi f)^{2}\left(\tilde{m}_{X}^{2}-m_{s s}^{2}\right)}+\frac{1}{12(4 \pi f)^{2}} \ln \left(\frac{m_{\pi}^{2}}{\mu^{2}}\right)\left[3 m_{\pi}^{2}-\frac{3 \tilde{\Delta}_{j u}^{2}\left(\tilde{m}_{X}^{2}+m_{\pi}^{2}\right)}{\tilde{m}_{X}^{2}-m_{\pi}^{2}}+\frac{\tilde{\Delta}_{j u}^{4} \tilde{m}_{X}^{2}}{\left(\tilde{m}_{X}^{2}-m_{\pi}^{2}\right)^{2}}-\frac{4 \tilde{\Delta}_{r s}^{2} m_{\pi}^{2}}{\left(\tilde{m}_{X}^{2}-m_{\pi}^{2}\right)\left(m_{s s}^{2}-m_{\pi}^{2}\right)}\right] \\
& -\frac{\tilde{m}_{X}^{2}}{12(4 \pi f)^{2}} \ln \left(\frac{\tilde{m}_{X}^{2}}{\mu^{2}}\right)\left[9-\frac{6 \tilde{\Delta}_{j u}^{2}}{\tilde{m}_{X}^{2}-m_{\pi}^{2}}+\frac{\tilde{\Delta}_{j u}^{4}}{\left(\tilde{m}_{X}^{2}-m_{\pi}^{2}\right)^{2}}+\frac{\tilde{\Delta}_{r s}^{2}\left(4\left(m_{K}^{2}-m_{\pi}^{2}\right)+6\left(m_{s s}^{2}-\tilde{m}_{X}^{2}\right)\right)}{\left(\tilde{m}_{X}^{2}-m_{s s}^{2}\right)^{2}}\right. \\
& \left.-\frac{2 \tilde{\Delta}_{j u}^{2} \tilde{\Delta}_{r s}^{2}\left(2 m_{s s}^{2}-m_{\pi}^{2}-\tilde{m}_{X}^{2}\right)}{\left(\tilde{m}_{X}^{2}-m_{s s}^{2}\right)^{2}\left(\tilde{m}_{X}^{2}-m_{\pi}^{2}\right)}\right]+\frac{1}{6(4 \pi f)^{2}} \ln \left(\frac{m_{s s}^{2}}{\mu^{2}}\right)\left[3 m_{s s}^{2}+\frac{\tilde{\Delta}_{r s}^{2}\left(3 m_{s s}^{4}+2\left(m_{K}^{2}-m_{\pi}^{2}\right) \tilde{m}_{X}^{2}-3 m_{s s}^{2} \tilde{m}_{X}^{2}\right)}{\left(\tilde{m}_{X}^{2}-m_{s s}^{2}\right)^{2}}\right. \\
& \left.\left.-\frac{\tilde{\Delta}_{j u}^{2} \tilde{\Delta}_{r s}^{2}\left(2 m_{s s}^{4}-\tilde{m}_{X}^{2}\left(m_{s s}^{2}+m_{\pi}^{2}\right)\right.}{\left(\tilde{m}_{X}^{2}-m_{s s}^{2}\right)^{2}\left(m_{s s}^{2}-m_{\pi}^{2}\right)}\right]\right\} .
\end{aligned}
$$

The two important things to note are that the additive lattice spacing modifications to the decay constants are the same and also that at this order, they can be absorbed into a redefinition of the Lagrangian parameter, $f$.

We can then use these formulas to estimate the size of the corrections to the recent determination of $L_{5}(\mu)$ by NPLQCD [23]. Thus, we form the ratio

$$
\Delta\left(\frac{f_{K}}{f_{\pi}}\right)=\frac{\left.\frac{f_{K}}{f_{\pi}}\right|_{M A}-\left.\frac{f_{K}}{f_{\pi}}\right|_{\mathrm{QCD}}}{\left.\frac{f_{K}}{f_{\pi}}\right|_{\mathrm{QCD}}},
$$

where, using Eqs. (C1) and (C2), and the tuning used in Ref. [23] which was to set the valence-valence meson masses equal to the taste- $\xi_{5}$ sea-sea mesons, we have 


$$
\begin{aligned}
\left.\frac{f_{K}}{f_{\pi}}\right|_{\mathrm{MA}}-\left.\frac{f_{K}}{f_{\pi}}\right|_{\mathrm{QCD}}= & \frac{m_{\pi}^{2}+a^{2} \Delta_{\mathrm{Mix}}}{\left(4 \pi f_{\pi}\right)^{2}} \ln \left(\frac{m_{\pi}^{2}+a^{2} \Delta_{\mathrm{Mix}}}{\mu^{2}}\right)-\frac{m_{\pi}^{2}}{\left(4 \pi f_{\pi}\right)^{2}} \ln \left(\frac{m_{\pi}^{2}}{\mu^{2}}\right)-\frac{m_{K}^{2}+a^{2} \Delta_{\mathrm{Mix}}}{\left(4 \pi f_{\pi}\right)^{2}} \ln \left(\frac{m_{K}^{2}+a^{2} \Delta_{\mathrm{Mix}}}{\mu^{2}}\right) \\
& +\frac{m_{K}^{2}}{2\left(4 \pi f_{\pi}\right)^{2}} \ln \left(\frac{m_{K}^{2}}{\mu^{2}}\right)-\frac{3}{4(4 \pi)^{2}}\left[\frac{m_{\eta}^{2}+a^{2} \Delta_{I}}{f_{\pi}^{2}} \ln \left(\frac{m_{\eta}^{2}+a^{2} \Delta_{I}}{\mu^{2}}\right)-\frac{m_{\eta}^{2}}{f_{\pi}^{2}} \ln \left(\frac{m_{\eta}^{2}}{\mu^{2}}\right)\right] \\
& -\frac{1}{2(4 \pi)^{2}}\left[\frac{m_{s s}^{2}+a^{2} \Delta_{\mathrm{Mix}}}{f_{\pi}^{2}} \ln \left(\frac{m_{s s}^{2}+a^{2} \Delta_{\mathrm{Mix}}}{\mu^{2}}\right)-\frac{m_{s s}^{2}}{f_{\pi}^{2}} \ln \left(\frac{m_{s s}^{2}}{\mu^{2}}\right)\right] \\
& -\left(\frac{a^{2} \Delta_{I}}{f_{\pi}^{2}}\right) \frac{1}{12(4 \pi)^{2}}\left\{3+\frac{4\left(m_{K}^{2}-m_{\pi}^{2}\right)}{m_{s s}^{2}-m_{\eta}^{2}-a^{2} \Delta_{I}}+\frac{3\left(m_{\eta}^{2}+a^{2} \Delta_{I}+m_{\pi}^{2}\right)}{m_{\eta}^{2}+a^{2} \Delta_{I}-m_{\pi}^{2}} \ln \left(\frac{m_{\pi}^{2}}{\mu^{2}}\right)\right. \\
& -\frac{2\left(3 m_{s s}^{4}-\left(m_{\eta}^{2}+a^{2} \Delta_{I}\right)\left(3 m_{s s}^{2}-2 m_{K}^{2}+2 m_{\pi}^{2}\right)\right)}{\left(m_{s s}^{2}-m_{\eta}^{2}-a^{2} \Delta_{I}\right)^{2}\left(\frac{m_{s s}^{2}}{\mu^{2}}\right)} \\
& \left.+2\left(m_{\eta}^{2}+a^{2} \Delta_{I}\right) \ln \left(\frac{m_{\eta}^{2}+a^{2} \Delta_{I}}{\mu^{2}}\right)\left[\frac{3 m_{s s}^{2}-3\left(m_{\eta}^{2}+a^{2} \Delta_{I}\right)+2 m_{K}^{2}-2 m_{\pi}^{2}}{\left(m_{s s}^{2}-m_{\eta}^{2}-a^{2} \Delta_{I}\right)^{2}} \frac{3}{m_{\eta}^{2}+a^{2} \Delta_{I}-m_{\pi}^{2}}\right]\right\} \\
& +\left(\frac{a^{2} \Delta_{I}}{f_{\pi}^{2}}\right)^{2} \frac{1}{12(4 \pi)^{2}}\left\{\frac{f_{\pi}^{2}}{m_{\eta}^{2}+a^{2} \Delta_{I}-m_{\pi}^{2}}-\frac{2 f_{\pi}^{2}}{m_{\eta}^{2}+a^{2} \Delta_{I}-m_{s s}^{2}}+\ln \left(\frac{m_{\pi}^{2}}{\mu^{2}}\right)\left[\frac{f_{\pi}^{2}\left(m_{\eta}^{2}+a^{2} \Delta_{I}\right)}{\left(m_{\eta}^{2}+a^{2} \Delta_{I}-m_{\pi}^{2}\right)^{2}}\right.\right. \\
& \left.-\frac{4 f_{\pi}^{2} m_{\pi}^{2}}{\left(m_{\eta}^{2}+a^{2} \Delta_{I}-m_{\pi}^{2}\right)\left(m_{s s}^{2}-m_{\pi}^{2}\right)}\right]-\ln \left(\frac{m_{s s}^{2}}{\mu^{2}}\right) \frac{2 f_{\pi}^{2}\left(2 m_{s s}^{4}-\left(m_{\eta}^{2}+a^{2} \Delta_{I}\right)\left(m_{s s}^{2}+m_{\pi}^{2}\right)\right)}{\left(m_{s s}^{2}-m_{\pi}^{2}\right)\left(m_{\eta}^{2}+a^{2} \Delta_{I}-m_{s s}^{2}\right)^{2}} \\
& \left.-\frac{m_{\eta}^{2}+a^{2} \Delta_{I}}{f_{\pi}^{2}} \ln \left(\frac{m_{\eta}^{2}+a^{2} \Delta_{I}}{\mu^{2}}\right)\left[\frac{f_{\pi}^{4}}{\left(m_{\eta}^{2}+a^{2} \Delta_{I}-m_{\pi}^{2}\right)^{2}}+\frac{2 f_{\pi}^{4}\left(m_{\eta}^{2}+a^{2} \Delta_{I}+m_{\pi}^{2}-2 m_{s s}^{2}\right)}{\left(m_{\eta}^{2}+a^{2} \Delta_{I}-m_{\pi}^{2}\right)\left(m_{\eta}^{2}+a^{2} \Delta_{I}-m_{s s}^{2}\right)^{2}}\right]\right\} .
\end{aligned}
$$

\section{APPENDIX D: $\pi^{+} \pi^{+}$SCATTERING}

For completeness, we provide the formulas for the $I=2$ $\pi \pi$ scattering length determined in both $\mathrm{MA} \chi \mathrm{PT}$ and $\mathrm{PQ} \chi \mathrm{PT}$ in Ref. [34], for both two and three flavors of sea quark.

\section{Two sea quark flavors, $m_{\pi} a_{\pi \pi}^{I=2}$}

$$
\begin{aligned}
m_{\pi} a_{\pi \pi}^{I=2}= & -\frac{m_{\pi}^{2}}{8 \pi f_{\pi}^{2}}\left\{1+\frac{m_{\pi}^{2}}{\left(4 \pi f_{\pi}\right)^{2}}\left[3 \ln \left(\frac{m_{\pi}^{2}}{\mu^{2}}\right)-1\right.\right. \\
& \left.\left.-l_{\pi \pi}^{I=2}(\mu)\right]-\frac{m_{\pi}^{2}}{\left(4 \pi f_{\pi}\right)^{2}} \frac{\tilde{\Delta}_{j u}^{4}}{6 m_{\pi}^{4}}\right\} .
\end{aligned}
$$

\section{Three sea quark flavors, $m_{\pi} a_{\pi \pi}^{I=2}$}

$$
\begin{aligned}
m_{\pi} a_{\pi \pi}^{I=2}= & -\frac{m_{\pi}^{2}}{8 \pi f_{\pi}^{2}}\left\{1+\frac{m_{\pi}^{2}}{\left(4 \pi f_{\pi}\right)^{2}}\left[3 \ln \left(\frac{m_{\pi}^{2}}{\mu^{2}}\right)-1\right.\right. \\
& \left.+\frac{1}{9} \ln \left(\frac{\tilde{m}_{X}^{2}}{\mu^{2}}\right)+\frac{1}{9}-32(4 \pi)^{2} L_{\pi \pi}^{I=2}(\mu)\right] \\
& +\frac{m_{\pi}^{2}}{\left(4 \pi f_{\pi}\right)^{2}}\left[-\frac{\tilde{\Delta}_{j u}^{4}}{6 m_{\pi}^{4}}\right. \\
& \left.\left.+\sum_{n=1}^{4}\left(\frac{\tilde{\Delta}_{j u}^{2}}{m_{\pi}^{2}}\right)^{n} \mathcal{F}_{n}\left(m_{\pi}^{2} / \tilde{m}_{X}^{2}\right)\right]\right\},
\end{aligned}
$$

where the functions $\mathcal{F}_{n}(y)$ are given by

$$
\begin{aligned}
& \mathcal{F}_{1}(y)=-\frac{2 y}{9(1-y)^{2}}[5(1-y)+(3+2 y) \ln (y)], \\
& \mathcal{F}_{2}(y)=\frac{2 y}{3(1-y)^{3}}[(1-y)(1+3 y)+y(3+y) \ln (y)], \\
& \mathcal{F}_{3}(y)=\frac{y}{9(1-y)^{4}}\left[(1-y)\left(1-7 y-12 y^{2}\right)-2 y^{2}(7+2 y) \ln (y)\right], \\
& \mathcal{F}_{4}(y)=-\frac{y^{2}}{54(1-y)^{5}}\left[(1-y)\left(1-8 y-17 y^{2}\right)-6 y^{2}(3+y) \ln (y)\right] .
\end{aligned}
$$

\section{APPENDIX E: $\boldsymbol{K}^{+} \boldsymbol{K}^{+}$SCATTERING}

The $I=1 K K$ and $I=3 / 2 K \pi$ scattering lengths involve rather lengthy expressions. Therefore, we introduce the following notation to make the answers more presentable: 


$$
m_{K}=k m_{\pi}, \quad \tilde{\Delta}_{j u}=\delta_{j u} m_{\pi}, \quad \tilde{\Delta}_{r s}=\delta_{r s} m_{\pi} . \text { (E1) } \quad C_{K}=2,
$$

The $I=1 K K$ scattering length is given by Eq. (30),

$$
\begin{aligned}
m_{K} a_{K K}^{I=1}= & -\frac{m_{K}^{2}}{8 \pi f_{K}^{2}}\left\{1+\frac{m_{K}^{2}}{\left(4 \pi f_{K}\right)^{2}}\left[C_{\pi} \ln \left(\frac{m_{\pi}^{2}}{\mu^{2}}\right)\right.\right. \\
& +C_{K} \ln \left(\frac{m_{K}^{2}}{\mu^{2}}\right)+C_{X} \ln \left(\frac{\tilde{m}_{X}^{2}}{\mu^{2}}\right)+C_{s s} \ln \left(\frac{m_{s s}^{2}}{\mu^{2}}\right) \\
& \left.\left.+C_{0}-32(4 \pi)^{2} L_{K K}^{I=1}\right]\right\},
\end{aligned}
$$

where

$$
\begin{aligned}
& C_{X}=-\frac{8\left(2-2 k^{2}+\delta_{j u}^{2}-\delta_{r s}^{2}\right)^{2}}{9\left(2-2 k^{2}+\delta_{j u}^{2}+2 \delta_{r s}^{2}\right)^{3}\left(4 k^{2}-4+\delta_{j u}^{2}+2 \delta_{r s}^{2}\right)^{3}}\left\{8\left(k^{2}-1\right)^{3}\left(20 k^{2}-11\right)\right. \\
& +4\left(k^{2}-1\right)^{2}\left(152 k^{2}-53\right) \delta_{r s}^{2}+12\left(38 k^{4}-61 k^{2}+23\right) \delta_{r s}^{4}+80\left(k^{2}-1\right) \delta_{r s}^{6}-8 \delta_{r s}^{8} \\
& +\delta_{j u}^{2}\left[14\left(k^{2}-1\right)^{2}\left(1+8 k^{2}\right)-24\left(5 k^{4}-4-k^{2}\right) \delta_{r s}^{2}-\left(312 k^{2}-132\right) \delta_{r s}^{4}-112 \delta_{r s}^{6}\right] \\
& \left.-\delta_{j u}^{4}\left[33\left(2 k^{4}-k^{2}-1\right)+\left(210 k^{2}-138\right) \delta_{r s}^{2}+102 \delta_{r s}^{4}\right]-\delta_{j u}^{6}\left(17 k^{2}-26+22 \delta_{r s}^{2}\right)+\delta_{j u}^{8}\right\}, \\
& C_{s s}=\frac{\delta_{r s}^{2}}{\left(k^{2}-1\right)^{3}\left(2-2 k^{2}+\delta_{j u}^{2}+2 \delta_{r s}^{2}\right)^{3}}\left\{8\left(k^{2}-1\right)^{4}\left(7 k^{2}-3\right)-4\left(k^{2}-1\right)^{3}\left(4 k^{2}-1\right) \delta_{r s}^{2}+4\left(k^{2}-1\right)^{2}\left(2 k^{2}-1\right) \delta_{r s}^{4}\right. \\
& -\delta_{j u}^{2}\left[4\left(k^{2}-1\right)^{3}\left(17 k^{2}-7\right)+2\left(k^{2}-1\right)^{2}\left(4 k^{2}-3\right) \delta_{r s}^{2}-4 k^{2}\left(k^{2}-1\right) \delta_{r s}^{4}\right]-\delta_{j u}^{6}\left(3 k^{4}-4 k^{2}+1+k^{2} \delta_{r s}^{2}\right) \\
& \left.+\delta_{j u}^{4}\left[2\left(k^{2}-1\right)^{2}\left(13 k^{2}-5\right)+2\left(5 k^{4}-7 k^{2}+2\right) \delta_{r s}^{2}-2 k^{2} \delta_{r s}^{4}\right]\right\} \text {, } \\
& C_{0}=\frac{2}{9\left(k^{2}-1\right)^{2}\left(4 k^{2}-4+\delta_{j u}^{2}+2 \delta_{r s}^{2}\right)^{2}\left(2-2 k^{2}+\delta_{j u}^{2}+2 \delta_{r s}^{2}\right)^{2}}\left\{-448\left(k^{2}-1\right)^{6}+1120\left(k^{2}-1\right)^{5} \delta_{r s}^{2}\right. \\
& +912\left(k^{2}-1\right)^{4} \delta_{r s}^{4}-152\left(k^{2}-1\right)^{3} \delta_{r s}^{6}-136\left(k^{2}-1\right)^{2} \delta_{r s}^{8}+\delta_{j u}^{8}\left[8\left(k^{2}-1\right)^{2}+18\left(k^{2}-1\right) \delta_{r s}^{2}+9 \delta_{r s}^{4}\right] \\
& -\delta_{j u}^{2}\left[112\left(k^{2}-1\right)^{5}-48\left(k^{2}-1\right)^{4} \delta_{r s}^{2}+876\left(k^{2}-1\right)^{3} \delta_{r s}^{4}+608\left(k^{2}-1\right)^{2} \delta_{r s}^{6}+72\left(k^{2}-1\right) \delta_{r s}^{8}\right] \\
& +\delta_{j u}^{4}\left[480\left(k^{2}-1\right)^{4}-96\left(k^{2}-1\right)^{3} \delta_{r s}^{2}-330\left(k^{2}-1\right)^{2} \delta_{r s}^{4}+36\left(k^{2}-1\right) \delta_{r s}^{6}+36 \delta_{r s}^{8}\right] \\
& \left.-\delta_{j u}^{6}\left[172\left(k^{2}-1\right)^{3}+140\left(k^{2}-1\right)^{2} \delta_{r s}^{2}-72\left(k^{2}-1\right) \delta_{r s}^{4}-36 \delta_{r s}^{6}\right]\right\} \text {. }
\end{aligned}
$$

\section{APPENDIX F: $\boldsymbol{K}^{+} \boldsymbol{\pi}^{+}$SCATTERING}

The $K \pi$ scattering length at $I=3 / 2$ is given by

$$
\begin{aligned}
\mu_{K \pi} a_{K \pi}^{I=3 / 2}= & -\frac{\mu_{K \pi}^{2}}{4 \pi f_{K} f_{\pi}}\left[1-\frac{32 m_{K} m_{\pi}}{f_{K} f_{\pi}} L_{\pi \pi}^{I=2}(\mu)\right. \\
& \left.+\frac{8\left(m_{K}-m_{\pi}\right)^{2}}{f_{K} f_{\pi}} L_{5}(\mu)\right] \\
& +\mu_{K \pi}\left[a_{v v}^{K \pi, 3 / 2}(\mu)+a_{v s}^{K \pi, 3 / 2}(\mu)\right],
\end{aligned}
$$

where $a_{v s}^{K \pi, 3 / 2}(\mu)$ is given in Eq. (43), which we repeat here for completeness,

$$
\begin{aligned}
\mu_{K \pi} a_{v s}^{K \pi, 3 / 2}(\mu)= & -\frac{\mu_{K \pi}^{2}}{4 \pi f_{K} f_{\pi}} \frac{1}{2(4 \pi)^{2} f_{K} f_{\pi}} \sum_{F=j, l, r} \\
& \times\left[C_{F s} \ln \left(\frac{\tilde{m}_{F s}^{2}}{\mu^{2}}\right)-C_{F d} \ln \left(\frac{\tilde{m}_{F d}^{2}}{\mu^{2}}\right)\right. \\
& \left.+4 m_{K} m_{\pi} J\left(\tilde{m}_{F d}^{2}\right)\right],
\end{aligned}
$$

and the coefficients $C_{F d, s}$ and the function $J(m)$ are defined in Eqs. (44)-(46). We reiterate that the $\ln \left(\mu^{2}\right)$ dependence in $a_{v s}^{K \pi, 3 / 2}(\mu)$ only depends upon the valence-valence meson masses, Eq. (47), as we argued in Sec. IIB. The valence-valence (and valence-ghost) contribution to the scattering length is given by

$$
\begin{aligned}
\mu_{K \pi} a_{v v}^{K \pi, 3 / 2}(\mu)= & \frac{\mu_{K \pi}^{2}}{4 \pi f_{K} f_{\pi}} \frac{m_{\pi}^{2}}{2(4 \pi)^{2} f_{K} f_{\pi}}\left[A_{\pi} \ln \left(\frac{m_{\pi}^{2}}{\mu^{2}}\right)\right. \\
& +A_{K} \ln \left(\frac{m_{K}^{2}}{\mu^{2}}\right)+A_{X} \ln \left(\frac{\tilde{m}_{X}^{2}}{\mu^{2}}\right) \\
& \left.+A_{s s} \ln \left(\frac{m_{s s}^{2}}{\mu^{2}}\right)+A_{\mathrm{tan}}+A_{0}\right] .
\end{aligned}
$$

We use the notation defined in Eq. (E1) to simplify the form of these coefficients. We find 


$$
\begin{aligned}
A_{\pi}= & \frac{1}{\left(k^{2}-1\right)^{3}\left(4 k^{2}-4+\delta_{j u}^{2}+2 \delta_{r s}^{2}\right)^{4}}\left\{8\left(k^{2}-1\right)^{2}\left(14 k-k^{2}-1\right)\left(2 k^{2}-2+\delta_{r s}^{2}\right)^{4}\right. \\
& +8 \delta_{j u}^{2}\left(k^{2}-1\right)\left(2 k^{2}-2+\delta_{r s}^{2}\right)^{3}\left[2 k^{6}+k^{4}\left(\delta_{r s}^{2}-1\right)+28 k^{3}+k^{2}\left(\delta_{r s}^{2}-2\right)-k\left(28-\delta_{r s}^{2}\right)+1\right] \\
& +2 \delta_{j u}^{4}\left(2 k^{2}-2+\delta_{r s}^{2}\right)^{2}\left[12 k^{8}+48 k^{7}+3 k^{6}\left(-5+2 \delta_{r s}^{2}\right)+32 k^{5} \delta_{r s}^{2}-9 k^{4}-2 k^{3}\left(72+21 \delta_{r s}^{2}-2 \delta_{r s}^{4}\right)\right. \\
& \left.+k^{2}\left(15-6 \delta_{r s}^{2}\right)+2 k\left(48+5 \delta_{r s}^{2}-\delta_{r s}^{4}\right)-3\right]+2 \delta_{j u}^{6}\left[4\left(k^{2}-1\right)^{3}\left(3 k^{4}+12 k^{3}+2 k^{2}+5 k-1\right)\right. \\
& \left.+2 \delta_{r s}^{2}\left(k^{2}-1\right)^{2}\left(6 k^{4}+32 k^{3}+5 k^{2}-2 k-1\right)+k \delta_{r s}^{4}\left(3 k^{5}+28 k^{4}-39 k^{2}-3 k+11\right)+2 k \delta_{r s}^{6}\left(2 k^{2}-1\right)\right] \\
& \left.+\delta_{j u}^{8}\left[2 k^{8}-k^{6}\left(3-\delta_{r s}^{2}\right)+2 k^{5}\left(5+2 \delta_{r s}^{2}\right)-k^{4}-k^{3}\left(20+5 \delta_{r s}^{2}-2 \delta_{r s}^{4}\right)+k^{2}\left(3-\delta_{r s}^{2}\right)+k\left(10+\delta_{r s}^{2}-\delta_{r s}^{4}\right)-1\right]\right\},
\end{aligned}
$$

$$
A_{K}=\frac{-2 k}{9(k-1)^{2}(k+1)}\left[40 k^{3}-26 k^{2}-4 k-10-(1+k)\left(2 \delta_{j u}^{2}+\delta_{r s}^{2}\right)\right]
$$

$$
\begin{aligned}
A_{s s}= & -\frac{1}{\left(k^{2}-1\right)^{3}\left(2-2 k^{2}+\delta_{j u}^{2}+2 \delta_{r s}^{2}\right)^{2}}\left\{2 ( k - 1 ) ^ { 2 } ( k + 1 ) ^ { 3 } \left[4 k^{7}-4 k^{6}+2 k^{5}\left(-5+\delta_{r s}^{2}\right)+2 k^{4}\left(3+5 \delta_{r s}^{2}\right)-2-\delta_{r s}^{4}\right.\right. \\
& \left.+2 k^{3}\left(4-\delta_{r s}^{2}\right)-8 k^{2}\left(\delta_{r s}^{2}+\delta_{r s}^{4}\right)-k\left(2+2 \delta_{r s}^{2}+5 \delta_{r s}^{4}\right)\right]-2 \delta_{j u}^{2}\left(k^{2}-1\right)\left[4 k^{8}+2 k^{6}\left(-7+\delta_{r s}^{2}\right)+4 k^{5}\left(-1+3 \delta_{r s}^{2}\right)\right. \\
& \left.+k^{4}\left(14+8 \delta_{r s}^{2}-\delta_{r s}^{4}\right)-2 k^{3}\left(-4+5 \delta_{r s}^{2}+\delta_{r s}^{4}\right)-k^{2}\left(2+10 \delta_{r s}^{2}+5 \delta_{r s}^{4}\right)-k\left(4+2 \delta_{r s}^{2}+3 \delta_{r s}^{4}\right)-2\right] \\
& +\delta_{j u}^{4}\left(2 k^{8}-k^{6}\left(7-\delta_{r s}^{2}\right)-k^{5}\left(2-6 \delta_{r s}^{2}\right)+k^{4}\left(7+4 \delta_{r s}^{2}\right)-k^{3}\left(-4+5 \delta_{r s}^{2}-2 \delta_{r s}^{4}\right)\right. \\
& \left.\left.-k^{2}\left(1+5 \delta_{r s}^{2}\right)-k\left(2+\delta_{r s}^{2}+\delta_{r s}^{4}\right)-1\right]\right\},
\end{aligned}
$$

$$
\begin{aligned}
A_{X}= & \frac{4\left(2-2 k^{2}+\delta_{j u}^{2}-\delta_{r s}^{2}\right)^{2}}{9(k-1)^{2}\left(2-2 k^{2}+\delta_{j u}^{2}+2 \delta_{r s}^{2}\right)^{2}\left(4 k^{2}-4+\delta_{j u}^{2}+2 \delta_{r s}^{2}\right)^{4}}\left\{-\delta_{j u}^{10} k-2 k \delta_{j u}^{8}\left(5 k^{2}+7 k-12+5 \delta_{r s}^{2}\right)\right. \\
& +2 \delta_{j u}^{6}\left[9 k^{6}-126 k^{5}+113 k^{4}+k^{3}\left(139-100 \delta_{r s}^{2}\right)+k^{2}\left(-167+64 \delta_{r s}^{2}\right)+k\left(41+36 \delta_{r s}^{2}-20 \delta_{r s}^{4}\right)-9\right] \\
& +2 \delta_{j u}^{4}\left[108 k^{8}-488 k^{7}+3 k^{6}\left(73+18 \delta_{r s}^{2}\right)+k^{5}\left(570-828 \delta_{r s}^{2}\right)+3 k^{4}\left(-47+274 \delta_{r s}^{2}\right)+81-54 \delta_{r s}^{2}\right. \\
& \left.-6 k^{3}\left(36-97 \delta_{r s}^{2}+62 \delta_{r s}^{4}\right)+3 k^{2}\left(-89-214 \delta_{r s}^{2}+112 \delta_{r s}^{4}\right)+k\left(134+66 \delta_{r s}^{2}+36 \delta_{r s}^{4}-40 \delta_{r s}^{6}\right)\right] \\
& +8 \delta_{j u}^{2}\left[108 k^{10}-56 k^{9}+2 k^{8}\left(-251+54 \delta_{r s}^{2}\right)-4 k^{7}\left(-87+86 \delta_{r s}^{2}\right)+3 k^{6}\left(232-23 \delta_{r s}^{2}+9 \delta_{r s}^{4}\right)\right. \\
& -6 k^{5}\left(82-104 \delta_{r s}^{2}+57 \delta_{r s}^{4}\right)+3 k^{4}\left(-124+13 \delta_{r s}^{2}+89 \delta_{r s}^{4}\right)+k^{3}\left(164-360 \delta_{r s}^{2}+273 \delta_{r s}^{4}-112 \delta_{r s}^{6}\right) \\
& \left.+k^{2}\left(124-159 \delta_{r s}^{2}-141 \delta_{r s}^{4}+88 \delta_{r s}^{6}\right)+k\left(36+80 \delta_{r s}^{2}-57 \delta_{r s}^{4}+24 \delta_{r s}^{6}-10 \delta_{r s}^{8}\right)-27\left(2-3 \delta_{r s}^{2}+\delta_{r s}^{4}\right)\right] \\
& +8\left(2 k^{2}-2+\delta_{r s}^{2}\right)^{2}\left[36 k^{8}-24 k^{7}+k^{6}\left(-85+18 \delta_{r s}^{2}\right)+k^{5}\left(40-28 \delta_{r s}^{2}\right)+k^{4}\left(71-46 \delta_{r s}^{2}\right)+9\right. \\
& \left.\left.-2 k^{3}\left(4-33 \delta_{r s}^{2}+8 \delta_{r s}^{4}\right)+k^{2}\left(-31+10 \delta_{r s}^{2}-4 \delta_{r s}^{4}\right)-2 k\left(4+\delta_{r s}^{2}-10 \delta_{r s}^{4}+2 \delta_{r s}^{6}\right)-18 \delta_{r s}^{2}\right]\right\},
\end{aligned}
$$

$$
\begin{aligned}
A_{0}= & \frac{-2}{9\left(k^{2}-1\right)^{2}\left(2-2 k^{2}+\delta_{j u}^{2}+2 \delta_{r s}^{2}\right)\left(4 k^{2}-4+\delta_{j u}^{2}+2 \delta_{r s}^{2}\right)^{3}}\left\{4096 k\left(k^{2}-1\right)^{6}+64 \delta_{r s}^{2}\left(k^{2}-1\right)^{5}\left(9 k^{2}+56 k+9\right)\right. \\
& +96 \delta_{r s}^{4}\left(k^{2}-1\right)^{4}\left(9 k^{2}-8 k+9\right)+16\left(k^{2}-1\right)^{3}\left(27 k^{2}-88 k+27\right) \delta_{r s}^{6}+8\left(k^{2}-1\right)^{2}\left(9 k^{2}-40 k+9\right) \delta_{r s}^{8} \\
& +\delta_{j u}^{2}\left[32\left(k^{2}-1\right)^{5}\left(9 k^{2}+14 k+9\right)+96 \delta_{r s}^{2}\left(k^{2}-1\right)^{4}\left(3 k^{2}-35 k+3\right)-24 \delta_{r s}^{4}\left(k^{2}-1\right)^{3}\left(9 k^{2}+166 k+9\right)\right. \\
& \left.-8 \delta_{r s}^{6}\left(k^{2}-1\right)^{2}\left(36 k^{2}+155 k+36\right)-72 \delta_{r s}^{8}\left(k^{4}+k^{3}-k-1\right)\right]+\delta_{j u}^{4}\left[48 k\left(k^{2}-1\right)^{4}\left(6 k^{2}+5\right)\right. \\
& +12 \delta_{r s}^{2}\left(k^{2}-1\right)^{3}\left(12 k^{3}-27 k^{2}-136 k-27\right)-6 \delta_{r s}^{4}\left(k^{2}-1\right)^{2}\left(36 k^{3}+63 k^{2}+230 k+63\right) \\
& \left.-36 \delta_{r s}^{6}\left(5 k^{5}+3 k^{4}+2 k^{3}-7 k-3\right)-36 \delta_{r s}^{8} k^{3}\right]-\delta_{j u}^{6}\left[2\left(k^{2}-1\right)^{3}\left(36 k^{3}+27 k^{2}+394 k+27\right)\right. \\
& \left.+2 \delta_{r s}^{2}\left(k^{2}-1\right)^{2}\left(108 k^{3}+63 k^{2}+323 k+63\right)+18 \delta_{r s}^{4}\left(9 k^{5}+3 k^{4}-4 k^{3}-5 k-3\right)+36 \delta_{r s}^{6} k^{3}\right] \\
& \left.-\delta_{j u}^{8}\left[\left(k^{2}-1\right)^{2}\left(36 k^{3}+9 k^{2}-22 k+9\right)+9\left(4 k^{5}+k^{4}-5 k^{3}+k-1\right) \delta_{r s}^{2}+9 k^{3} \delta_{r s}^{4}\right]\right\},
\end{aligned}
$$




$$
\begin{aligned}
A_{\tan }= & \frac{4 k}{(k-1)\left(2-2 k^{2}+\delta_{j u}^{2}+2 \delta_{r s}^{2}\right)^{2} \sqrt{(k-1)^{3}(k+1)}} \arctan \left(\frac{\sqrt{(k-1)^{3}(k+1)}}{k^{2}+k-1}\right)\left\{8(k-1)^{4}(k+1)^{3}+4 \delta_{r s}^{2}\left(k^{2}-1\right)^{2}\right. \\
& \left.+4 \delta_{r s}^{4}\left(k^{3}-2 k^{2}-k+2\right)-\delta_{j u}^{2}\left[8(k-1)^{3}(k+1)^{2}+4 \delta_{r s}^{2}\left(k^{2}-1\right)-2 \delta_{r s}^{4}\right]+\delta_{j u}^{4}\left[2(k-1)^{2}(k+1)+\delta_{r s}^{2}\right]\right\} \\
& -\frac{8 k\left(2-2 k^{2}+\delta_{j u}^{2}-\delta_{r s}^{2}\right)^{2} \sqrt{\left(8 k^{2}-12 k+4-\delta_{j u}^{2}-2 \delta_{r s}^{2}\right)\left(4 k^{2}-4+\delta_{j u}^{2}+2 \delta_{r s}^{2}\right)}}{9(k-1)^{2}\left(2-2 k^{2}+\delta_{j u}^{2}+2 \delta_{r s}^{2}\right)^{2}} \\
& \times \arctan \left(\frac{\sqrt{\left(8 k^{2}-12 k+4-\delta_{j u}^{2}-2 \delta_{r s}^{2}\right)\left(4 k^{2}-4+\delta_{j u}^{2}+2 \delta_{r s}^{2}\right)}}{4 k^{2}+6 k-4+\delta_{j u}^{2}+2 \delta_{r s}^{2}}\right) .
\end{aligned}
$$

[1] D. B. Kaplan, Phys. Lett. B 288, 342 (1992).

[2] Y. Shamir, Nucl. Phys. B406, 90 (1993).

[3] V. Furman and Y. Shamir, Nucl. Phys. B439, 54 (1995).

[4] R. Narayanan and H. Neuberger, Nucl. Phys. B412, 574 (1994).

[5] R. Narayanan and H. Neuberger, Phys. Rev. Lett. 71, 3251 (1993).

[6] R. Narayanan and H. Neuberger, Nucl. Phys. B443, 305 (1995).

[7] P. H. Ginsparg and K. G. Wilson, Phys. Rev. D 25, 2649 (1982).

[8] M. Luscher, Phys. Lett. B 428, 342 (1998).

[9] K. G. Wilson, Phys. Rev. D 10, 2445 (1974).

[10] J. B. Kogut and L. Susskind, Phys. Rev. D 11, 395 (1975).

[11] L. Susskind, Phys. Rev. D 16, 3031 (1977).

[12] C. W. Bernard and M. F. L. Golterman, Phys. Rev. D 49, 486 (1994).

[13] S. R. Sharpe, Phys. Rev. D 56, 7052 (1997).

[14] O. Bar, G. Rupak, and N. Shoresh, Phys. Rev. D 67, 114505 (2003).

[15] O. Bar, G. Rupak, and N. Shoresh, Phys. Rev. D 70, 034508 (2004).

[16] K. C. Bowler, B. Joo, R. D. Kenway, C. M. Maynard, and R. J. Tweedie (UKQCD Collaboration), J. High Energy Phys. 08 (2005) 003.

[17] F. D. R. Bonnet, R. G. Edwards, G. T. Fleming, R. Lewis, and D. G. Richards, (Lattice Hadron Physics) Phys. Rev. D 72, 054506 (2005).

[18] R. G. Edwards et al. (LHPC Collaboration), Phys. Rev. Lett. 96, 052001 (2006).

[19] S. R. Beane, P. F. Bedaque, K. Orginos, and M. J. Savage (NPLQCD Collaboration), Phys. Rev. D 73, 054503 (2006).

[20] S. R. Beane, P. F. Bedaque, K. Orginos, and M. J. Savage, Phys. Rev. Lett. 97, 012001 (2006).

[21] S. R. Beane, K. Orginos, and M.J. Savage, hep-lat/ 0604013.

[22] S. R. Beane, K. Orginos, and M. J. Savage, hep-lat/ 0605014.

[23] S. R. Beane, P. F. Bedaque, K. Orginos, and M. J. Savage, hep-lat/0606023.

[24] C. Alexandrou, T. Leontiou, J. W. Negele, and A. Tsapalis, hep-lat/0607030.

[25] S. R. Beane et al., Phys. Rev. D 74, 114503 (2006).

[26] O. Bar, K. Jansen, S. Schaefer, L. Scorzato, and A. Shindler, hep-lat/0609039.

[27] C. W. Bernard et al., Phys. Rev. D 64, 054506 (2001); http://qcd.nersc.gov/.

[28] K. Orginos and D. Toussaint (MILC Collaboration), Phys. Rev. D 59, 014501 (1998).

[29] K. Orginos, D. Toussaint, and R. L. Sugar (MILC Collaboration), Phys. Rev. D 60, 054503 (1999).

[30] O. Bar, C. Bernard, G. Rupak, and N. Shoresh, Phys. Rev. D 72, 054502 (2005).

[31] B. C. Tiburzi, Nucl. Phys. A761, 232 (2005).

[32] M. Golterman, T. Izubuchi, and Y. Shamir, Phys. Rev. D 71, 114508 (2005).

[33] B. C. Tiburzi, Phys. Rev. D 72, 094501 (2005).

[34] J.-W. Chen, D. O'Connell, R.S. Van de Water, and A. Walker-Loud, Phys. Rev. D 73, 074510 (2006).

[35] T. B. Bunton, F. J. Jiang, and B. C. Tiburzi, Phys. Rev. D 74, 034514 (2006).

[36] C. Aubin, J. Laiho, and R. S. V. de Water, hep-lat/0609009.

[37] J. Gasser and H. Leutwyler, Ann. Phys. (N.Y.) 158, 142 (1984).

[38] J. Gasser and H. Leutwyler, Nucl. Phys. B250, 465 (1985).

[39] S.R. Sharpe and R.S. Van de Water, Phys. Rev. D 71, 114505 (2005).

[40] D. O'Connell, hep-lat/0609046.

[41] C. Aubin et al. (MILC Collaboration), Phys. Rev. D 70, 114501 (2004).

[42] P. H. Damgaard and K. Splittorff, Phys. Rev. D 62, 054509 (2000).

[43] S. R. Sharpe, Proc. Sci. LAT2006 (2006) 022 [hep-lat/ 0610094].

[44] C. W. Bernard and M. F. L. Golterman, Phys. Rev. D 46, 853 (1992).

[45] S. R. Sharpe, Phys. Rev. D 46, 3146 (1992).

[46] C. W. Bernard and M. F. L. Golterman, Phys. Rev. D 53, 476 (1996).

[47] E. B. Gregory, A. C. Irving, C. C. McNeile, S. Miller, and Z. Sroczynski, Proc. Sci., LAT2005 (2006) 027 [hep-lat/ 0510066].

[48] S. Prelovsek, Phys. Rev. D 73, 014506 (2006). 
[49] C. W. Bernard, C. DeTar, Z. Fu, and S. Prelovsek, hep-lat/ 0610031.

[50] S. R. Sharpe and N. Shoresh, Phys. Rev. D 62, 094503 (2000).

[51] S. R. Sharpe and N. Shoresh, Phys. Rev. D 64, 114510 (2001).

[52] S. R. Sharpe and R. J. Singleton, Phys. Rev. D 58, 074501 (1998).

[53] K. Symanzik, Nucl. Phys. B226, 205 (1983).

[54] K. Symanzik, Nucl. Phys. B226, 187 (1983).

[55] B. Sheikholeslami and R. Wohlert, Nucl. Phys. B259, 572 (1985).

[56] R. Frezzotti, P. A. Grassi, S. Sint, and P. Weisz (Alpha Collaboration), J. High Energy Phys. 08 (2001) 058.

[57] S. R. Sharpe and J.M.S. Wu, Phys. Rev. D 71, 074501 (2005).

[58] C. Aubin and C. Bernard, Phys. Rev. D 68, 034014 (2003).

[59] M. Knecht, B. Moussallam, J. Stern, and N. H. Fuchs, Nucl. Phys. B457, 513 (1995).

[60] S. R. Sharpe and R. S. Van de Water, Phys. Rev. D 69, 054027 (2004).

[61] J.-W. Chen and M. J. Savage, Phys. Rev. D 65, 094001 (2002).

[62] S. R. Beane and M. J. Savage, Nucl. Phys. A709, 319 (2002).

[63] S. R. Beane and M. J. Savage, Phys. Rev. D 67, 054502 (2003).

[64] A. Walker-Loud, Nucl. Phys. A747, 476 (2005).

[65] B. C. Tiburzi and A. Walker-Loud, Nucl. Phys. A748, 513 (2005).

[66] W. Detmold and C. J.D. Lin, Phys. Rev. D 71, 054510 (2005).

[67] B. C. Tiburzi and A. Walker-Loud, Nucl. Phys. A764, 274 (2006).

[68] D. O'Connell and M. J. Savage, Phys. Lett. B 633, 319 (2006).

[69] W. Detmold, B. C. Tiburzi, and A. Walker-Loud, Phys. Rev. D 73, 114505 (2006).

[70] A. Walker-Loud, Ph.D. thesis, University of Washington, 2006, hep-lat/0608010.

[71] W.-J. Lee and S.R. Sharpe, Phys. Rev. D 60, 114503 (1999).

[72] D. B. Renner et al. (LHP Collaboration), Nucl. Phys. B, Proc. Suppl. 140, 255 (2005).
[73] R. G. Edwards et al. (LHPC Collaboration), Proc. Sci., LAT2005 (2006) 056 [hep-lat/0509185].

[74] P. F. Bedaque, I. Sato, and A. Walker-Loud, Phys. Rev. D 73, 074501 (2006).

[75] H. W. Hamber, E. Marinari, G. Parisi, and C. Rebbi, Nucl. Phys. B225, 475 (1983).

[76] M. Luscher, Commun. Math. Phys. 105, 153 (1986).

[77] M. Luscher, Nucl. Phys. B354, 531 (1991).

[78] Silas Beane (private communication).

[79] J. Stern, H. Sazdjian, and N. H. Fuchs, Phys. Rev. D 47, 3814 (1993).

[80] G. Colangelo, J. Gasser, and H. Leutwyler, Phys. Lett. B 488, 261 (2000).

[81] G. Colangelo, J. Gasser, and H. Leutwyler, Phys. Rev. Lett. 86, 5008 (2001).

[82] G. Colangelo, J. Gasser, and H. Leutwyler, Nucl. Phys. B603, 125 (2001).

[83] J. Bijnens, G. Colangelo, G. Ecker, J. Gasser, and M.E. Sainio, Phys. Lett. B 374, 210 (1996).

[84] J. Bijnens, G. Colangelo, G. Ecker, J. Gasser, and M. E. Sainio, Nucl. Phys. B508, 263 (1997).

[85] J. Bijnens, G. Colangelo, and P. Talavera, J. High Energy Phys. 05 (1998) 014.

[86] B. Ananthanarayan, G. Colangelo, J. Gasser, and H. Leutwyler, Phys. Rep. 353, 207 (2001).

[87] S. Pislak et al. (BNL-E865), Phys. Rev. Lett. 87, 221801 (2001).

[88] S. Pislak et al., Phys. Rev. D 67, 072004 (2003).

[89] DIRAC Collaboration, http://dirac.web.cern.ch/DIRAC/ future.html.

[90] V. Bernard, N. Kaiser, and U. G. Meissner, Nucl. Phys. B357, 129 (1991).

[91] V. Bernard, N. Kaiser, and U. G. Meissner, Phys. Rev. D 43, R2757 (1991).

[92] B. Kubis and U.-G. Meissner, Nucl. Phys. A699, 709 (2002).

[93] B. Kubis and U.-G. Meissner, Phys. Lett. B 529, 69 (2002).

[94] J. Bijnens, P. Dhonte, and P. Talavera, J. High Energy Phys. 05 (2004) 036

[95] C. Bernard, Phys. Rev. D 73, 114503 (2006).

[96] J. Bijnens and N. Danielsson, Phys. Rev. D 74, 054503 (2006).

[97] R. G. Edwards et al., hep-lat/0610007. 